\title{
Spatio-temporal Models of Lymphangiogenesis in Wound Healing
}

\author{
Arianna Bianchi, Kevin J. Painter, Jonathan A. Sherratt
}

2016

\begin{abstract}
Several studies suggest that one possible cause of impaired wound healing is failed or insufficient lymphangiogenesis, that is the formation of new lymphatic capillaries. Although many mathematical models have been developed to describe the formation of blood capillaries (angiogenesis), very few have been proposed for the regeneration of the lymphatic network. Lymphangiogenesis is a markedly different process from angiogenesis, occurring at different times and in response to different chemical stimuli. Two main hypotheses have been proposed: 1) lymphatic capillaries sprout from existing interrupted ones at the edge of the wound in analogy to the blood angiogenesis case; 2) lymphatic endothelial cells first pool in the wound region following the lymph flow and then, once sufficiently populated, start to form a network. Here we present two PDE models describing lymphangiogenesis according to these two different hypotheses. Further, we include the effect of advection due to interstitial flow and lymph flow coming from open capillaries. The variables represent different cell densities and growth factor concentrations, and where possible the parameters are estimated from biological data. The models are then solved numerically and the results are compared with the available biological literature.
\end{abstract}

\section{Introduction}

The lymphatic system first came to the anatomists' attention with Hippocrates' mention of lymph nodes in his $5^{\text {th }}$ century BC work On Joints 112]. Later, the Roman physician Rufus of Ephesus identified the axillary, inguinal and mesenteric nodes and the thymus in the $1^{\text {st }}-2^{\text {nd }}$ century $\mathrm{AD} 67$. The earliest reference to lymphatic vessels is attributed to the anatomist Herophilus, who lived in Alexandria in the $3^{\text {rd }}$ century BC; he described the lymphatics as "absorptive veins" 22, 105. This rudimentary knowledge of the lymphatic system was lost during the Middle Ages, until Gabriele Falloppio re-discovered lymphatic capillaries in the mid- $16^{\text {th }}$ century 16 . Since then, there has been a steady but slow increase in our awareness of the "second" circulatory system of our body (see 2 for an account of immunology's priority disputes in the $17^{\text {th }}$ and $18^{\text {th }}$ centuries). Major impetus to study the lymphatic system came only in the 1990s, after the discovery of a suitable lymphatic marker that allowed quantifiable observation of lymphatic dynamics 18,77 . Lymphatic research is still a current trend in biomedicine and a source of sensational new discoveries, such as the 2015 finding of lymphatic vessels in the central nervous system [58].

An impetus for studying lymphatic regeneration is provided by recent biological studies that propose lymphangiogenesis as a major target for the treatment of non-healing wounds: functional lymphangiogenesis is nowadays regarded as a crucial factor in wound healing [17, 46, 77, 113] and delayed or failed lymphatic regeneration (such as that observed in diabetic patients) constitutes a major cause of impairment to wound healing $3,66,87$.

Interest in lymphatics is therefore not just a mere scientific curiosity: their importance as pressure regulators in tissues and, moreover, as vectors of the immune response has been emphasised in recent decades, particularly in the context of wound healing 17, 43, 46. The healing of a skin wound is a complex process consisting of different overlapping phases that, if well orchestrated by the organism, lead to the restoration of the skin and vasculature to a healthy, functional condition. Unfortunately, this delicate sequence of events can fail to proceed to full healing in diabetic or aged patients [3, 45, 98]; indeed, if the organism response to infection is insufficient, wound healing does not proceed through all normal stages, halting at the inflammation stage and resulting in a chronic wound 10,79 .

Non-healing wounds constitute a major health problem, seriously affecting the patient's quality of life and accounting for approximately $3 \%$ of all health care expenses in the UK 24 81 . Being the main mediators of the immune response, lymphatics seem to significantly contribute to healing [77\|113] and it has been observed that failed lymphangiogenesis correlates with impaired wound healing [3, 66, 87]. However, little is known about the actual mechanisms involved in the lymphangiogenic process, in contrast with the (blood) angiogenic case 5: 18].

Mathematical modelling potentially provides an alternative, powerful tool to back up experimental observations, generate a better understanding of wound healing lymphangiogenesis and identify potential clinical targets. Here we build upon our ODE model presented in 7 to address the spatial elements of lymphangiogenesis, specifically focussing on modelling two different hypotheses proposed to describe the exact lymphangiogenesis mechanism. 


\section{Biological background}

\subsection{Wound healing}

For educational purposes, wound healing is usually presented as a sequence of four different (overlapping) phases, namely:

1. Hemostasis: Shortly after injury, a blood clot is formed as a result of the interaction between blood and the extracellular matrix; the clot stops the bleeding and provides a scaffold for cells and chemicals that will consequently contribute to the healing process.

2. Inflammation: Substances activated during hemostasis attract leukocytes, inflammatory cells which clean the wound from debris and pathogens and secrete chemicals which promote the evolution of the system to the next phase.

3. Proliferation: The chemicals released during inflammation enhance the growth and aggregation of the surrounding cells, restoring different tissue functions and elements such as the blood and lymphatic networks; the regeneration of blood and lymphatic vessels is named (blood) angiogenesis and lymphangiogenesis, respectively. In this phase, the blood clot is slowly substituted by a "temporary dermis" called granulation tissue. In parallel with these processes, the rapid proliferation and migration of epidermal cells causes this outer layer of the skin to re-form.

4. Remodelling: Finally, the granulation tissue is slowly replaced by normal skin tissue; this stage can take up to two years to be completed.

For further details about wound healing we refer to 94 for normal cutaneous wound healing, and to 95 for an account of chronic wound dynamics.

\subsection{Sprouting versus self-organising lymphangiogenesis}

The lymphatic system is a circulatory system responsible for mediating the immune response of the body and maintaining the physiological pressure in tissues by draining excess liquid. It is mainly constituted of vessels and lymph nodes. Lymphatic vessel walls are made of so-called lymphatic endothelial cells (LECs); contrary to the blood case, lymphatic capillaries are very thin and are formed of a single layer of LECs.

To date, little is known about the biological events taking place during lymphangiogenesis and different hypotheses have been proposed by biologists. Although important reviews on the subject such as 74,99 state that lymphangiogenesis "occurs primarily by sprouting from pre-existing vessels", in a fashion which resembles the (blood) angiogenic case, recent experiments suggest that this may not be correct, at least not in some specific experimental settings 5 85]. In [5] it is stated that lymphangiogenesis "can occur both by recruitment of isolated lymphatic islands to a connected network and by filopodial sprouting". Similarly, in [85 it is reported that in an adult mouse tail wound model, LECs migrate as single cells into the wound space and later connect to each other forming vessel structures (see Figure 1). According to the authors of [85, single LEC migration following the lymph/interstitial flow would explain why lymphatic vessel regeneration appears to happen in this direction (from left to right in the figure). Comparative reviews of lymphangiogenesis and (blood) angiogenesis can be found in 1, 57, 97.

\subsection{Interstitial versus lymph flow}

Interstitial flow is a fluid flow induced by dynamic stresses and pressure gradients through the extracellular matrix. It is generally slower than fluid flow inside vessels, because of the resistance of the extracellular matrix components; nonetheless, interstitial flow has recently been shown to play an important role in many processes, including cell migration. Such effects can be purely mechanical, for example by "pushing" on the cell, or can act indirectly by shifting the distribution of chemicals in the surroundings of the cell. A review of the effects of interstitial flow on cell biology can be found in 86 .

In recent years, a number of studies have investigated the role of interstitial flow on lymphangiogenesis, mainly through the formation of concentration gradients of pro-lymphangiogenic factors. In particular, in 8 the authors propose that interstitial flow, enhanced by the lymph flow resulting from interrupted lymphatic vessels, can direct wound healing lymphangiogenesis by transporting LECs into the wound space and creating gradients in chemicals (such as vascular endothelial growth factor - VEGF) which stimulate LEC growth and chemotaxis. However, the relative role of interstitial and lymph flow on capillary regeneration has yet to be investigated in depth; therefore, it is not clear which of the two takes on the greatest importance. In fact, although interstitial flow is slower than the flux of the lymph coming from interrupted capillaries, the former persists after wound closure, while the latter is more localised to open capillaries and stops once the lymphatic network has been restored. 


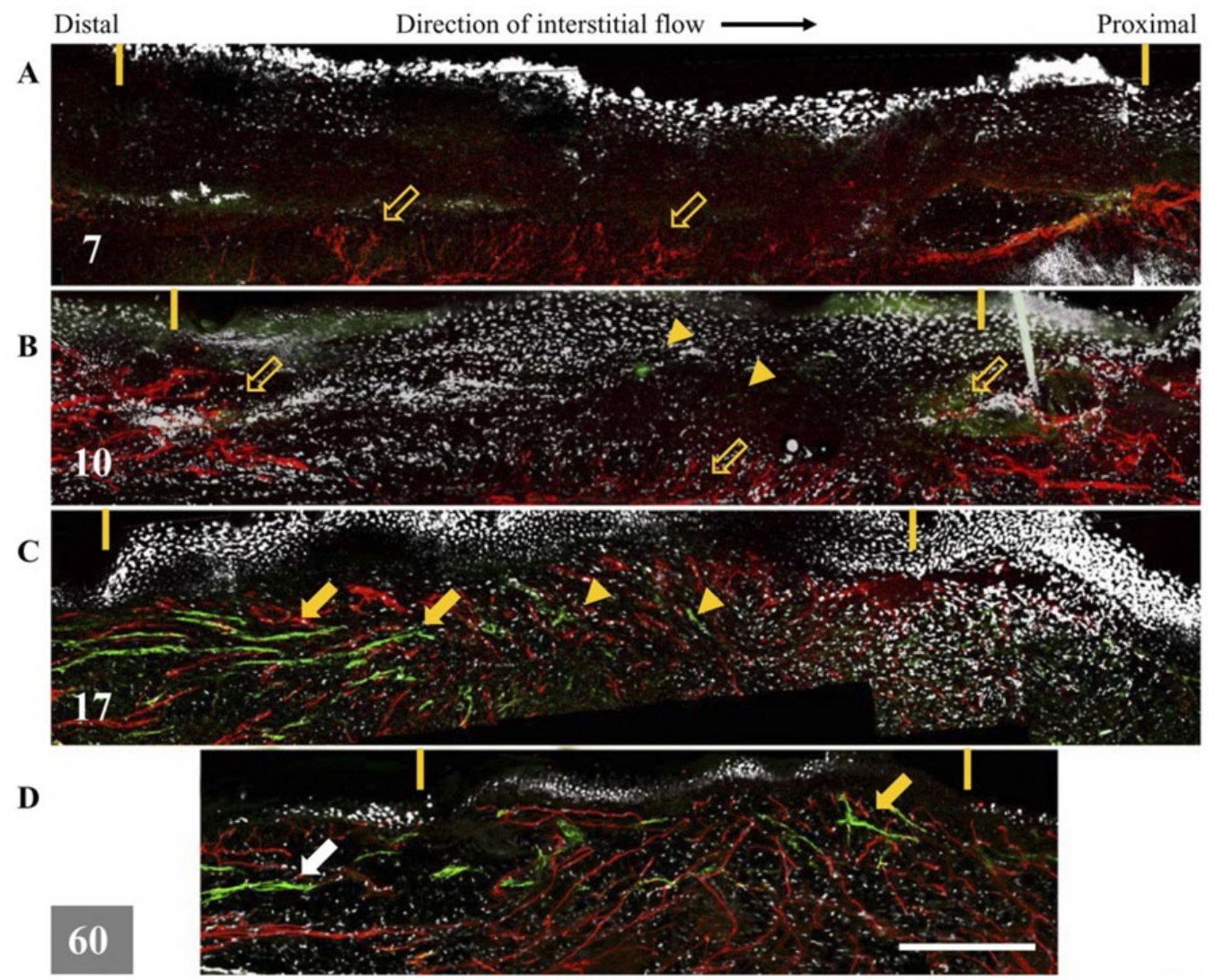

Figure 1: In the photo, taken from [85, Figure 2], one observes blood and lymphatic vessel regeneration in the tail of an adult mouse; lymphangiogenesis appears to occur in the direction of the interstitial flow. The different photos refer to different times after wounding: A was taken at day 7, B at day 10 , C at day 17 and $\mathrm{D}$ at day 60 . The yellow dashes mark the regenerating region (note its overall contraction over time); the red colour indicates blood vessels, while LEC presence is highlighted by green colour. The open arrows signal how blood vessels seem to sprout from deeper vessels, while other arrows point out LEC organisation at day 17 after a higher LEC density is reached; arrowheads indicate single LECs migrating towards the proximal side of the wound. Scale bar in $\mathrm{D}=300 \mu \mathrm{m}$.

\section{Mathematical Modelling}

\subsection{Review of lymphatic-related models}

Contrary to the blood angiogenesis case, modelling literature about lymphangiogenesis is relatively immature and sparse, and mostly refers to tumour-induced lymphangiogenesis (see for instance [32]). To the authors' knowledge, the only models addressing lymphangiogenesis in wound healing are [84, which focuses on the mechanical elements that lend the lymphatic network its characteristic shape (at least in the mouse tail), and our previous work [7, which we are going to extend here. A recent review of mathematical models of vascular network formation is [89], where indeed the imbalance between blood angio- and lymphangio-genesis modelling is manifest.

A number of models have been produced by the bioengineering community, describing specific mechanical features of lymphatic physiology; in particular, mechanics of contracting lymph valves have been presented in $33,41,62,68,82$. A brief review of engineering models proposed in the lymphatic context can be found in 65 .

Very few attempts have been made to specifically model the effect of flow on capillary regeneration, although one interesting example is 31], where the authors use a convection-diffusion model to analyse the effects of flow on matrix-binding protein gradients.

\section{$3.2 \quad$ Model targets}

The model hereby presented aims to investigate the following questions about wound healing lymphangiogenesis:

- which hypothesis (self-organising or sprouting) offers a better explanation for the lymphangiogenesis mechanics? 
- what are the relative contributions of interstitial and lymph flow on the lymphangiogenic process?

- how does the initial wounded state impact on lymphatic regeneration?

\subsection{Model variables and domain}

In the following, we propose two similar but distinct PDE models to describe the two different theories advanced by biologists to explain lymphangiogenesis in wound healing (see Section 2.2). We will refer to them as the "self-organising" hypothesis (O) and the "sprouting" hypothesis (S).

For both cases, we consider the following basic dynamics: immediately after injury, transforming growth factor- $\beta$ (TGF- $\beta$ ) is activated and chemotactically attracts macrophages to the wound, which in turn secrete VEGF which induces capillary regeneration acting on either LECs (in the self-organising case) or capillary tips (in the sprouting case). The variables included in the models are summarised in Table 1, where they are reported together with their names and units.

\begin{tabular}{cccc}
\hline VARIABLE & MODEL & QUANTITY & UNITS \\
\hline$T(t, x)$ & O,S & active TGF- $\beta$ concentration & $\mathrm{pg} \cdot \mathrm{mm}^{-3}$ \\
$M(t, x)$ & O,S & macrophage density & cells $\cdot \mathrm{mm}^{-3}$ \\
$V(t, x)$ & $\mathrm{O}, \mathrm{S}$ & VEGF concentration & $\mathrm{pg} \cdot \mathrm{mm}^{-3}$ \\
$L(t, x)$ & $\mathrm{O}$ & lymphatic endothelial cell density & $\mathrm{cells} \cdot \mathrm{mm}^{-3}$ \\
$E(t, x)$ & $\mathrm{S}$ & lymphatic capillary end (tip) density & $\mathrm{cells} \cdot \mathrm{mm}^{-3}$ \\
$C(t, x)$ & $\mathrm{O}, \mathrm{S}$ & lymphatic capillary density & $\mathrm{cells} \cdot \mathrm{mm}^{-3}$ \\
\hline
\end{tabular}

Table 1: A summary of the model variables.

We consider a $1 \mathrm{D}$ space variable $x$ that varies between $-\varepsilon$ and $\ell+\varepsilon$; this interval includes the wound space of length $\ell$ and a portion $\varepsilon$ of healthy tissue on its edges. This kind of domain describes a narrow cut, where at every point we average chemical and cell densities over the depth of the wound. We take the increasing- $x$ direction to be that of lymph flow (and interstitial flow). A schematic of the model domain is shown in Figure 2 .

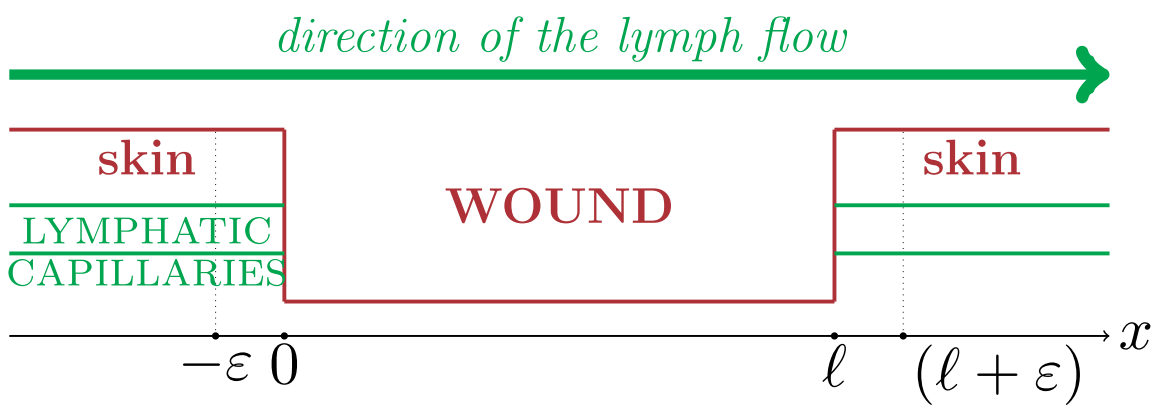

Figure 2: The model 1D domain.

\subsection{Advection velocity and open capillaries}

The models incorporate an advection term for the majority of variables that accounts for the effect of flow on the lymphatic regeneration process. In biological references (such as 8]) it is not clear whether flow is mainly a result of lymph fluid exiting the interrupted capillaries, or the "normal" interstitial flow. We hence investigate the relative contribution from these two components by considering an advection term motivated as follows.

In general, interstitial flow does not have a constant direction. However, for simplicity, here we will assume that both lymph and interstitial flow occur in the increasing direction of $x$ (from left to right in Figure 22); this reflects what is observed in the wound healing experimental setting of [8], which we take as a reference for model comparison. We assume the interstitial flow to be constant and present across the full tissue, reflecting its persistent nature in healthy tissues. On the other hand, the contribution due to leaking lymphatic capillaries is assumed to depend specifically on the density of open capillaries $C_{o p}$ and we assume a linear dependence for simplicity. However, since we do not know the precise contribution of each element to the total advection, we introduce a single "weight" parameter $\xi, 0 \leq \xi \leq 1$, which can be varied. Specifically, the advection velocities for chemicals and cells, $\lambda^{\text {chem }}$ and $\lambda^{\text {cell }}$ respectively, will be taken to be of the forms

$$
\begin{aligned}
\lambda^{\text {chem }}\left(C_{o p}\right) & =\xi \cdot\left(\lambda_{1}^{\text {chem }} \cdot C_{o p}\right)+(1-\xi) \cdot \lambda_{2}^{\text {chem }} \text { and } \\
\lambda^{\text {cell }}\left(C_{o p}\right) & =\xi \cdot\left(\lambda_{1}^{\text {cell }} \cdot C_{o p}\right)+(1-\xi) \cdot \lambda_{2}^{\text {cell }}
\end{aligned}
$$


where $0 \leq \xi \leq 1$ and $\lambda_{1}^{\text {chem }}, \lambda_{2}^{\text {chem }}, \lambda_{1}^{\text {cell }}, \lambda_{2}^{\text {cell }}$ are four parameters to be determined. In Appendix A.4 we estimate the values of $\lambda_{1}^{\text {chem }}$ and $\lambda_{2}^{\text {chem }}$, while corresponding parameters for cells are assumed to be significantly smaller, since advective cell velocity is likely to be smaller due to the higher environmental friction. A value of $\xi=0$ corresponds to purely interstitial flow advection, while $\xi=1$ represents advection due entirely to lymphatic flow.

To quantify the open capillary density, we assume that as the "cut" in capillary density $C$ becomes steeper (and thus $|\partial C / \partial x| \rightarrow+\infty$ ), more capillaries are open and the open capillary density will increase towards its maximum possible value of $C$, which would correspond to all capillaries being open. We therefore define the open capillary density $C_{o p}$ as

$$
C_{o p}\left(C, \frac{\partial C}{\partial x}\right)=\frac{|\partial C / \partial x|}{\eta_{0}+|\partial C / \partial x|} \cdot C
$$

where $\eta_{0}$ is a parameter for whose estimation no relevant experimental data were found. See Figure 3 for a plot of 3 .
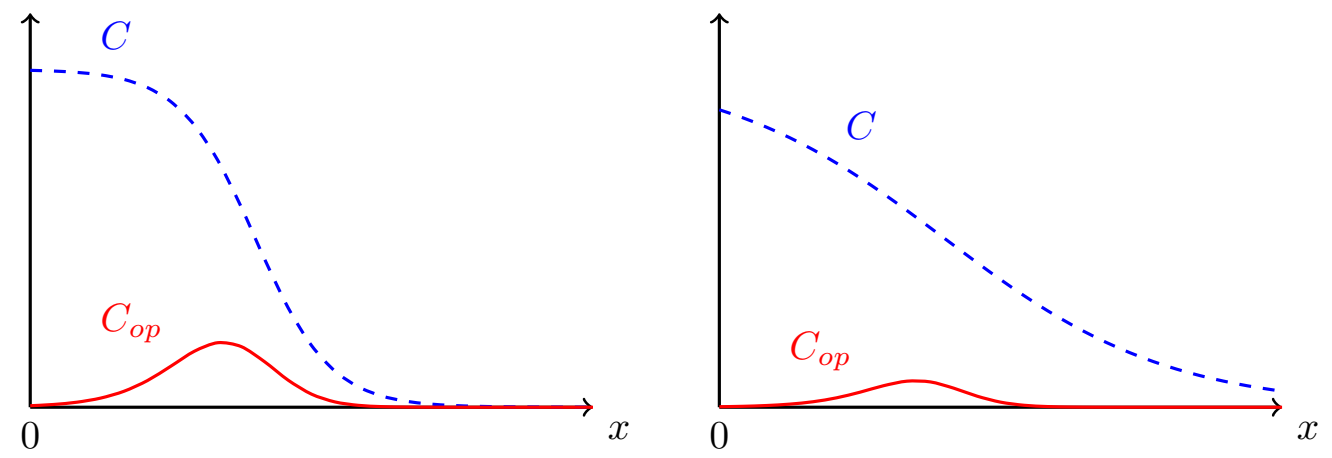

Figure 3: Plots of $C_{o p}$ (solid red) for different steepness of $C$ (dashed blue).

\subsection{Self-organising hypothesis}

Under this hypothesis, single LECs migrate into the wound and start to self-organise into capillary structures only after reaching a certain threshold density $L^{*}$. This case represents the direct extension of the ODE model developed in [7] and the variable and parameter names have been kept as consistent with [7] as possible.

\section{(Active) TGF- $\beta$ equation}

The differential equation describing active TGF- $\beta$ concentration has the following form:

$$
\begin{gathered}
\begin{array}{c}
\text { change in TGF- } \beta \\
\text { concentration }
\end{array} \\
\text { advection }
\end{gathered} \quad+\text { activation }- \text { decay }-\quad \begin{gathered}
\text { internalisation by } \\
\text { macrophages. }
\end{gathered}
$$

Of these terms, the following three are assumed to have standard forms:

$$
\text { Diffusion: } D_{T} \frac{\partial^{2} T}{\partial x^{2}} \quad, \quad \text { Decay: } d_{1} T \quad, \quad \text { Internalisation: } \gamma_{1} T M
$$

and advection will be taken to be $-\partial / \partial x\left(\lambda^{\text {chem }}\left(C_{o p}\right) \cdot T\right)$, with velocity $\lambda^{\text {chem }}\left(C_{o p}\right)$ as defined in $(1)$.

Concerning the activation, we consider a constant amount of latent TGF- $\beta$ in the skin $T_{L}$ [91, 100, which is increased by macrophage production at rate $r_{1}[50]$. This latent form of TGF- $\beta$ is activated by macrophages 21, 36, 75, 100 and by the enzymes (mainly plasmin) present in the blood clot, which is mainly composed of platelets [37, 44, 49, (for a review of TGF- $\beta$ activation see [100]). Therefore, we take the following activation term:

$$
\underbrace{\left[a_{m} M+a_{p} p(C)\right]}_{\begin{array}{c}
\text { activation by macro- } \\
\text {-phages \& plasmin }
\end{array}} \cdot \underbrace{\left[T_{L}+r_{1} M\right]}_{\text {latent TGF- } \beta} .
$$

The $C$-dependent quantity $p$ is an estimate of plasmin presence in the wound, which is proportional to the platelet mass. In fact, although activation of platelet-released TGF- $\beta$ is still poorly understood, it seems that plasmin, while degrading the blood clot, activates the latent TGF- $\beta$ contained in the platelets [37]. We assume that the plasmin level is proportional to the wound space which is not occupied by capillaries; this is motivated 
by the fact that capillary presence can be considered as a measure of the healing stage of the wound 11 When capillary density gets close to its equilibrium (healthy state) value $C^{e q}$ (say $90 \%$ of it), the plasmin-induced TGF- $\beta$ activation switches to zero. We will thus take

$$
p(C)=\left\{\begin{array}{cl}
-\frac{\psi}{9 / 10 \cdot C} C+\psi & \text { if } C \leq\left(C^{e q} \cdot 9 / 10\right) \\
0 & \text { if } C \geq\left(C^{e q} \cdot 9 / 10\right) .
\end{array}\right.
$$

\section{Macrophage equation}

The following scheme will be considered for macrophage dynamics:

\begin{tabular}{|c|c|c|c|c|c|c|c|c|}
\hline \multirow[t]{2}{*}{$\begin{array}{l}\text { change in } \\
\text { macrophage } \\
\text { density }\end{array}$} & $=$ & $\begin{array}{l}\text { random movement } \\
\text { and advection }\end{array}$ & + & $\begin{array}{c}\text { chemotaxis by } \\
\text { TGF- } \beta\end{array}$ & + & $\begin{array}{l}\text { constant } \\
\text { source }\end{array}$ & + & $\begin{array}{l}\text { influx from } \\
\text { open } \\
\text { capillaries }\end{array}$ \\
\hline & - & $\begin{array}{l}\text { removal and } \\
\text { differentiation }\end{array}$ & - & crowding effect. & & & & \\
\hline
\end{tabular}

Macrophages are assumed to move randomly with diffusion coefficient $\mu_{M}$, while their advection will be modelled by the term $-\frac{\partial}{\partial x}\left(\lambda^{\text {cell }}\left(C_{o p}\right) \cdot M\right)$, with $\lambda^{\text {cell }}\left(C_{o p}\right)$ as discussed in Section 3.4 .

For the chemotaxis term, we first point out that only a fraction $\alpha$ of the monocytes that are chemoattracted by TGF- $\beta$ differentiate into (inflammatory) macrophages 63, 107]. Therefore, the term describing macrophage chemotaxis up TGF- $\beta$ gradients will have the form

$$
-\alpha \chi_{1} \frac{\partial}{\partial x}\left(\frac{M}{1+\omega M} \cdot \frac{\partial T / \partial x}{1+\eta_{1}|\partial T / \partial x|}\right)
$$

where the macrophage velocity $\frac{1}{1+\omega M} \cdot \frac{\partial T / \partial x}{1+\eta_{1}|\partial T / \partial x|}$ decreases as cell density increases (as in 103,104 ) and is bounded as $|\partial T / \partial x| \rightarrow \infty$. The presence of a constant source $s_{M}$ (from the bottom of the wound) is justified by the observation that the macrophage equilibrium in unwounded skin is nonzero [110].

The introduction of an influx term is motivated by the fact that macrophages are "pumped out" from interrupted capillaries 8, 85, and into the wound. We consider the following form for the influx term:

$$
\varphi_{1}\left(C_{o p}, \frac{\partial C}{\partial x}\right)=C_{o p} \cdot \zeta_{1}\left(\frac{\partial C}{\partial x}\right)
$$

where $C_{o p}$ was introduced in $(3)$ and $\zeta_{1}$ is defined as

$$
\zeta_{1}\left(\frac{\partial C}{\partial x}\right)=\left\{\begin{array}{cl}
\phi_{1} & \text { if } \partial C / \partial x<0 \\
0 & \text { otherwise } .
\end{array}\right.
$$

In (6) $\phi_{1}$ is a parameter estimated in Appendix A.7. The Heaviside form of $\zeta_{1}$ is due to the influx only occurring from the open lymphatic capillaries on the side of the wound from which lymph fluid flows (see Figure 2).

The removal term includes (inflammatory) macrophage death, differentiation into repair macrophages and reintroduction into the vascular system, with the latter being proportional to the capillary density. Thus, we take the removal term to be $\left(d_{2}+\rho C\right) M$. We also include a crowding effect through the term $-\frac{M+L+C}{k_{1}} \cdot M$.

\section{VEGF equation}

For VEGF we assume the following dynamics:

$\begin{array}{lcccc}\begin{array}{c}\text { change in } \\ \text { VEGF } \\ \text { concentration }\end{array} & \begin{array}{c}\text { diffusion and } \\ \text { advection }\end{array} & + & \text { constant source } & +\begin{array}{c}\text { production by } \\ \text { macrophages }\end{array} \\ & - & \text { decay } & - & \text { internalisation by } \\ & \text { LECs. }\end{array}$

VEGF diffusion is modelled via the standard term $D_{V} \frac{\partial^{2} V}{\partial x^{2}}$ and advection by $-\frac{\partial}{\partial x}\left(\lambda^{\text {chem }}\left(C_{o p}\right) \cdot V\right)$ where $\lambda^{\text {chem }}\left(C_{o p}\right)$ is the expression defined in (1). The constant source is called $s_{V}$, while the production term will be $r_{3} M$ and the decay $d_{3} V$. Internalisation is assumed to be linearly dependent on LEC density and the corresponding term will consequently be $\gamma_{2} V L$.

\footnotetext{
${ }^{1}$ An alternative approach would be to consider fibroblasts instead of capillaries here, but the introduction of a new variable and consequently a new equation does not seem to be worthwhile, since capillary presence is a good indication of the healing state of the wound.
} 


\section{LEC equation}

The equation describing the presence of LECs in the wound consists of the following terms:

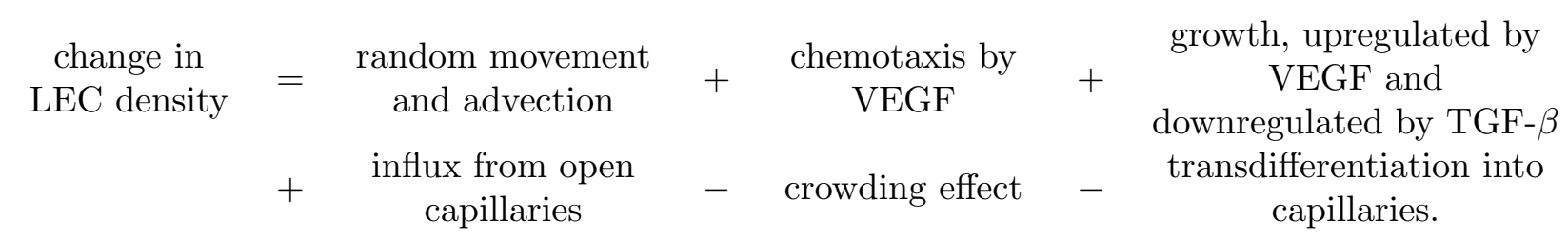

Again, random cell movement is modelled via a diffusion term $\mu_{L} \partial^{2} L / \partial x^{2}$ and the advection is taken to be $-\frac{\partial}{\partial x}\left(\lambda^{\text {cell }}(C) \cdot L\right)$.

LECs are chemoattracted by VEGF 6, 99], and the chemotaxis term is assumed to be of a similar form to that used to describe macrophage chemotaxis:

$$
-\chi_{2} \frac{\partial}{\partial x}\left(\frac{L}{1+\omega L} \cdot \frac{\partial V / \partial x}{1+\eta_{2}|\partial V / \partial x|}\right) .
$$

LEC growth is upregulated by VEGF 6, 111, 116, and downregulated by TGF- $\beta$,71, 96]:

$$
\left(c_{1}+\frac{V}{c_{2}+c_{3} V}\right)\left(\frac{1}{1+c_{4} T}\right) L .
$$

LECs are "pumped out" from the interrupted capillaries in a similar manner to macrophages, but also result (with less intensity) from interrupted capillaries downstream of the lymph flow. The influx term this time takes the form:

$$
\varphi_{2}\left(C_{o p}, \frac{\partial C}{\partial x}\right)=C_{o p} \cdot \zeta_{2}\left(\frac{\partial C}{\partial x}\right)
$$

where $C_{o p}$ is the density of open capillaries as in $(3)$ and $\zeta_{2}$ is defined as

$$
\zeta_{2}\left(\frac{\partial C}{\partial x}\right)= \begin{cases}\phi_{2}^{+} & \text {if } \partial C / \partial x<0 \\ \phi_{2}^{-} & \text {if } \partial C / \partial x>0\end{cases}
$$

where $\phi_{2}^{+}>\phi_{2}^{-}$.

LECs cannot grow excessively due to crowding, which is taken into account via the term $-\frac{(M+L+C)}{k_{2}} \cdot L$. When LECs have locally sufficiently populated the wound (i.e. when their density exceeds a threshold $L^{*}[8,85]$ ) they are assumed to self-organise into capillaries at a rate which is increased by the presence of VEGF 80 :

$$
\sigma(L, C) \cdot\left(\delta_{1}+\delta_{2} V\right) L
$$

where

$$
\sigma(L, C)= \begin{cases}1 & \text { if } L+C \geq L^{*} \\ 0 & \text { if } L+C<L^{*}\end{cases}
$$

\section{Lymphatic capillary equation}

After LECs have occupied enough of the wound space, they coalesce into a capillary network; also, they undergo remodelling, which we model via a logistic term. Thus, the $C$-equation will be

$$
\underbrace{\sigma(L, C) \cdot\left(\delta_{1}+\delta_{2} V\right) L}_{\text {source }}+\underbrace{c_{5}\left(1-\frac{C}{k_{3}}\right) C}_{\text {remodelling }} .
$$

Observe that no advection term is present here, since capillary structures are collections of cells attached to each other and thus are more resistant to the interstitial flows.

\section{Full system - "self-organising" hypothesis}

The full system of equations in the "self-organising" hypothesis is therefore given by 


$$
\begin{aligned}
\frac{\partial T}{\partial t}= & D_{T} \frac{\partial^{2} T}{\partial x^{2}}-\frac{\partial}{\partial x}\left(\lambda^{\text {chem }}\left(C_{o p}\right) \cdot T\right)+\left[a_{m} M+a_{p} p(C)\right] \cdot\left[T_{L}+r_{1} M\right] \\
& -d_{1} T-\gamma_{1} T M \\
\frac{\partial M}{\partial t}= & \mu_{M} \frac{\partial^{2} M}{\partial x^{2}}-\frac{\partial}{\partial x}\left(\lambda^{\text {cell }}\left(C_{o p}\right) \cdot M+\alpha \chi_{1} \frac{M}{1+\omega M} \cdot \frac{\partial T / \partial x}{1+\eta_{1}|\partial T / \partial x|}\right) \\
& +s_{M}+\varphi_{1}\left(C_{o p}, \frac{\partial C}{\partial x}\right)-\left(d_{2}+\rho C\right) M-\frac{M+L+C}{k_{1}} M, \\
\frac{\partial V}{\partial t}= & D_{V} \frac{\partial^{2} V}{\partial x^{2}}-\frac{\partial}{\partial x}\left(\lambda^{c h e m}\left(C_{o p}\right) \cdot V\right)+s_{V}+r_{3} M-d_{3} V-\gamma_{2} V L \\
\frac{\partial L}{\partial t}= & \mu_{L} \frac{\partial^{2} L}{\partial x^{2}}-\frac{\partial}{\partial x}\left(\lambda^{c e l l}\left(C_{o p}\right) \cdot L+\chi_{2} \frac{L}{1+\omega L} \cdot \frac{\partial V / \partial x}{1+\eta_{2}|\partial V / \partial x|}\right) \\
& +\left(c_{1}+\frac{V}{c_{2}+c_{3} V}\right)\left(\frac{1}{1+c_{4} T}\right) L+\varphi_{2}\left(C_{o p}, \frac{\partial C}{\partial x}\right) \\
& -\frac{M+L+C}{k_{2}} L-\sigma(L, C) \cdot\left(\delta_{1}+\delta_{2} V\right) L, \\
\frac{\partial C}{\partial t}= & \sigma(L, C) \cdot\left(\delta_{1}+\delta_{2} V\right) L+c_{5}\left(1-\frac{C}{k_{3}}\right) C,
\end{aligned}
$$

where $\lambda^{\text {chem }}$ is defined in (1), $\lambda^{\text {cell }}$ in (2), $p$ in (4), $\varphi_{1}$ in (5), $\varphi_{2}$ in (7) and $\sigma$ in (9). Parameters, initial and boundary conditions are discussed in Sections 3.7 and 3.8 respectively. See Figure 4 for a summary of the fluxes included in the model.

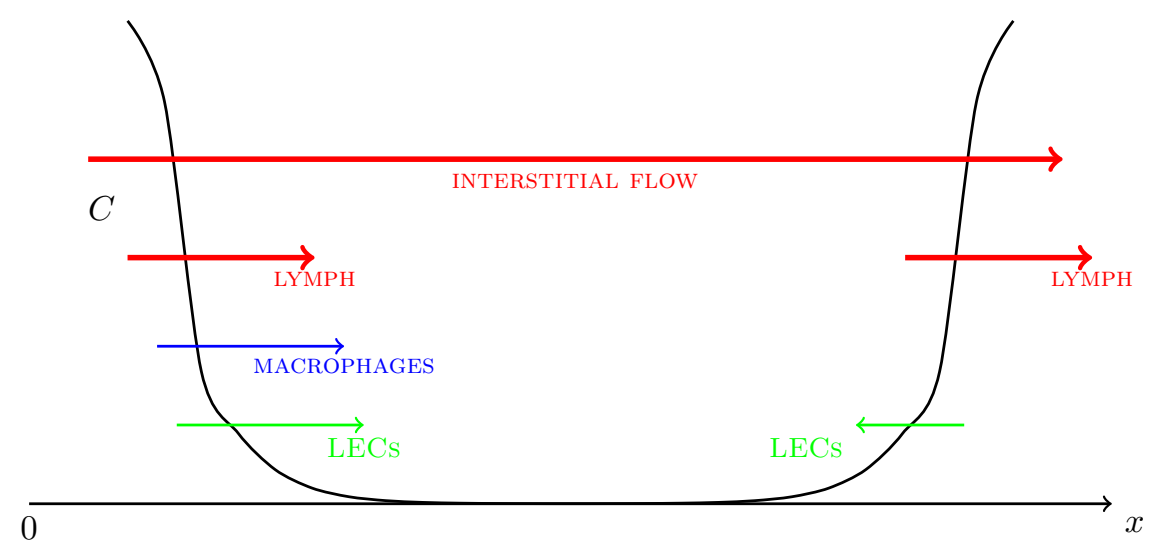

Figure 4: A summary of the fluxes included in the model: capillaries; fluid fluxes; macrophage influx; LEC influx (only in $\mathrm{O}$ ).

\subsection{Sprouting hypothesis}

Here, instead of LECs we consider capillary tip density E. Capillary tips are attached to the vessel ends and therefore, contrary to LECs, are not subject to advection. As we will see, the introduction of this variable is necessary in order to model directed capillary growth in response to a gradient. Examples of mathematical models of blood angiogenesis (in wound healing and in tumours) which include the capillary tip variable can be found in $12,13,29,30,55,64,88$.

TGF- $\beta$, macrophage and VEGF equations are the same as in the self-organising case, except that in both the crowding term for $M$ and the $V$ internalisation term there is $E$ instead of $L$.

\section{Lymphatic capillary ends (tips) equation}

Capillary ends (or tips) are assumed to sprout from interrupted lymphatic capillaries, the density of which $\left(C_{o p}\right)$ was defined in (3). Tip growth is enhanced by VEGF and inhibited by TGF- $\beta$ and this is reflected by the following term, similar to the one used for LECs in the self-organising case:

$$
\left(c_{1}+\frac{V}{c_{2}+c_{3} V}\right)\left(\frac{1}{1+c_{4} T}\right) C_{o p} .
$$


Importantly, capillary ends move in the direction of the (positive) gradient of VEGF with an upper-bounded velocity, modelled by the term

$$
-\chi_{2} \frac{\partial}{\partial x}\left(E \cdot \frac{\partial V / \partial x}{1+\eta_{2}|\partial V / \partial x|}\right) .
$$

Finally, we assume that capillary tip death is due predominantly to overcrowding, and thus we include the removal term $-\frac{(M+E+C)}{k_{2}} \cdot E$.

\section{Lymphatic capillary equation}

New capillaries are formed continuously from the interrupted ones in the direction defined by their tips. This is modelled here according to the "snail trail" concept which was introduced in 25 for fungal colonies and which has been widely used in models of (blood) angiogenesis [30]: newly formed capillaries are laid after the sprouting tips, which therefore leave a sort of "track" behind.

Capillaries also undergo remodelling. Therefore, their dynamics are captured by the terms:

$$
\underbrace{\chi_{2}\left|E \cdot \frac{\partial V / \partial x}{1+\eta_{2}|\partial V / \partial x|}\right|}_{\text {sprouting }}+\underbrace{c_{5}\left(1-\frac{C}{k_{3}}\right) C}_{\text {remodelling }} .
$$

\section{Full system - "sprouting" hypothesis}

Thus, the full system for the "sprouting" hypothesis is

$$
\begin{aligned}
\frac{\partial T}{\partial t}= & D_{T} \frac{\partial^{2} T}{\partial x^{2}}-\frac{\partial}{\partial x}\left(\lambda^{\text {chem }}\left(C_{o p}\right) \cdot T\right)+\left[a_{m} M+a_{p} p(C)\right] \cdot\left[T_{L}+r_{1} M\right] \\
& -d_{1} T-\gamma_{1} T M, \\
\frac{\partial M}{\partial t}= & \mu_{M} \frac{\partial^{2} M}{\partial x^{2}}-\frac{\partial}{\partial x}\left(\lambda^{\text {cell }}\left(C_{o p}\right) \cdot M+\alpha \chi_{1} \frac{M}{1+\omega M} \cdot \frac{\partial T / \partial x}{1+\eta_{1}|\partial T / \partial x|}\right) \\
& +s_{M}+\varphi_{1}\left(C_{o p}, \frac{\partial C}{\partial x}\right)-\left(d_{2}+\rho C\right) M-\frac{M+E+C}{k_{1}} M, \\
\frac{\partial V}{\partial t}= & D_{V} \frac{\partial^{2} V}{\partial x^{2}}-\frac{\partial}{\partial x}\left(\lambda^{c h e m}\left(C_{o p}\right) \cdot V\right)+s_{V}+r_{3} M-d_{3} V-\gamma_{2} V E, \\
\frac{\partial E}{\partial t}= & \left(c_{1}+\frac{V}{c_{2}+c_{3} V}\right)\left(\frac{1}{1+c_{4} T}\right) C_{o p}-\chi_{2} \frac{\partial}{\partial x}\left(E \cdot \frac{\partial V / \partial x}{1+\eta_{2}|\partial V / \partial x|}\right) \\
& -\frac{M+E+C}{k_{2}} E, \\
\frac{\partial C}{\partial t}= & \chi_{2}\left|E \cdot \frac{\partial V / \partial x}{1+\eta_{2}|\partial V / \partial x|}\right|+c_{5}\left(1-\frac{C}{k_{3}}\right) C,
\end{aligned}
$$

where $\lambda^{\text {chem }}$ is defined in (1), $\lambda^{\text {cell }}$ in (2), $p$ in (4), $\varphi_{1}$ in $(5)$ and $C_{o p}$ in (3) (see Figure 4 for a summary of the fluxes of the model).

\subsection{Parameters}

All the model parameters are reported in Table 2. Many of the parameters were estimated previously in [7] and we refer to this source for details of their estimation. For the other parameters listed in Table 2 , the details of their estimation can be found in Appendix A.

\subsection{Initial and boundary conditions}

\section{Initial Conditions}

As initial time $t=0$ we take the moment of wounding, when little chemical or cell populations are assumed to have entered in the wound space. Specifically, we assume that at $t=0$ there are no LECs (for model O) or capillary tips (for S), while other variables can be present near the edges (recall our domain includes portions of healthy skin surrounding the wound). We will then take the following initial conditions:

$$
\begin{aligned}
\nu(0, x) & =a_{\nu} \cdot\left[1-\frac{\tanh (b x)+\tanh (b(-x+\ell))}{2}\right], \\
L(0, x) & =E(0, x)=0
\end{aligned}
$$




\begin{tabular}{|c|c|c|c|c|}
\hline PARAMETER & VALUE & UNITS & SOURCE & DETAILS \\
\hline$D_{T}$ & 2.76 & $\mathrm{~mm}^{2}$ day $^{-1}$ & 54,72 & \begin{tabular}{l|l} 
Appendix \\
\end{tabular} \\
\hline$\eta_{0}$ & $10^{4}$ & cells $\mathrm{mm}^{-4}$ & no data found & Appendix \\
\hline$\lambda_{1}^{\text {chem }}$ & $1.35 \times 10^{-2}$ & mmday $^{-1}$ & \begin{tabular}{|l|l|}
27 & 28 \\
\end{tabular} & Appendix \\
\hline$\lambda_{2}^{\text {chem }}$ & $8.64 \times 10^{2}$ & mmday $^{-1}$ & 86 & Appendix \\
\hline$a_{p}$ & $2.9 \times 10^{-2}$ & $\mathrm{~mm}^{3} \mathrm{pg}^{-1}$ day $^{-1}$ & 21 & 7 \\
\hline$\psi$ & $10^{5}$ & $\mathrm{pg} \mathrm{mm^{-3 }}$ & no data found & Appendix A \\
\hline$a_{m}$ & 0.45 & $\mathrm{~mm}^{3}$ cells $^{-1}$ day $^{-1}$ & \begin{tabular}{|l|l|}
36 & 75
\end{tabular} & 7 \\
\hline$T_{L}$ & 18 & $\mathrm{pg} \mathrm{mm^{-3 }}$ & $(76)$ & 7 \\
\hline$r_{1}$ & $3 \times 10^{-5}$ & pg cells ${ }^{-1}$ day $^{-1}$ & 50 & 7 \\
\hline$d_{1}$ & $5 \times 10^{2}$ & day $^{-1}$ & 47 & 7 \\
\hline$\gamma_{1}$ & $4.2 \times 10^{-3}$ & $\mathrm{~mm}^{3}$ cells $^{-1}$ day $^{-1}$ & $(115)$ & Appendix A \\
\hline$\mu_{M}$ & 0.12 & $\mathrm{~mm}^{2}$ day $^{-1}$ & $\overline{26}$ & Appendix \\
\hline$\lambda_{1}^{\text {cell }}$ & $1.35 \times 10^{-3}$ & mmday $^{-1}$ & estimated $\approx 0.1 \times \lambda_{1}^{\text {chem }}$ & Appendix \\
\hline$\lambda_{2}^{\text {cell }}$ & 86.4 & mmday $^{-1}$ & estimated $\approx 0.1 \times \lambda_{2}^{\text {chem }}$ & Appendix $\bar{A}$ \\
\hline$\alpha$ & 0.5 & 1 & 109 & 7 \\
\hline$\chi_{1}$ & $4 \times 10^{-2}$ & $\mathrm{~mm}^{5} \mathrm{pg}^{-1}$ day $^{-1}$ & 56 & Appendix A \\
\hline$\omega$ & $1.67 \times 10^{-6}$ & $\mathrm{~mm}^{3}$ cells $^{-1}$ & estimated $\approx 1 / k_{1}^{\text {old }}$ & Appendix $\bar{A}$ \\
\hline$\eta_{1}$ & 100 & $\mathrm{~mm}^{9} \mathrm{pg}^{-1}$ & no data found & Appendix \\
\hline$s_{M}$ & $8.6 \times 10^{2}$ & cells $\mathrm{mm}^{-3} \mathrm{day}^{-1}$ & $(110)$ & Appendix \\
\hline$\phi_{1}$ & $2.05 \times 10^{3}$ & day $^{-1}$ & 14,28 & Appendix \\
\hline$\beta$ & $5 \times 10^{-3}$ & 1 & 38 & 7 \\
\hline$r_{2}$ & 1.22 & day $^{-1}$ & 118 & 7 \\
\hline$d_{2}$ & 0.2 & day $^{-1}$ & 20 & 7 \\
\hline$\rho$ & $10^{-5}$ & day $^{-1}$ cells $^{-1}$ & 85 & 7 \\
\hline$k_{1}$ & $10^{5}$ & $\mathrm{~mm}^{3}$ cells $^{-1}$ & 118 & Appendix A \\
\hline$D_{V}$ & 2.4 & $\mathrm{~mm}^{2}$ day $^{-1}$ & $\overline{69}$ & Appendix $\overline{\mathrm{A}}$ \\
\hline$s_{V}$ & 1.94 & cells day $^{-1}$ & $(42,78)$ & 7 \\
\hline$r_{3}$ & $1.9 \times 10^{-3}$ & pg cells ${ }^{-1}$ day $^{-1}$ & $(51,90)$ & 7 \\
\hline$d_{3}$ & 11 & day $^{-1}$ & 52 & 7 \\
\hline$\gamma_{2}$ & $1.4 \times 10^{-3}$ & $\mathrm{~mm}^{3}$ cells $^{-1}$ day $^{-1}$ & 61 & 7 \\
\hline$\mu_{L}$ & 0.1 & $\mathrm{~mm}^{2}$ day $^{-1}$ & estimated $\approx \mu_{M}$ & Appendix $\mathrm{A}$ \\
\hline$c_{1}$ & 0.42 & day $^{-1}$ & 73 & 7 \\
\hline$c_{2}$ & 42 & day & 111 & 7 \\
\hline$c_{3}$ & 4.1 & $\mathrm{pg}$ day $\mathrm{mm}^{-3}$ & 111 & 7 \\
\hline$c_{4}$ & 0.24 & $\mathrm{~mm}^{3} \mathrm{pg}^{-1}$ & 71 & 7 \\
\hline$\chi_{2}$ & 0.173 & $\mathrm{~mm}^{5} \mathrm{pg}^{-1} \mathrm{day}^{-1}$ & 4 & Appendix A \\
\hline$\eta_{2}$ & 1 & $\mathrm{~mm}^{9} \mathrm{pg}^{-1}$ & no data found & Appendix \\
\hline$\phi_{2}^{+}$ & $10^{2}$ & day $^{-1}$ & no data found & Appendix \\
\hline$\phi_{2}^{-}$ & 1 & day $^{-1}$ & estimated to be $1 \%$ of $\phi_{2}^{+}$ & Appendix $\overline{\mathrm{A}}$ \\
\hline$k_{2}$ & $4.71 \times 10^{5}$ & cells day $\mathrm{mm}^{-3}$ & 73 & 7 \\
\hline$L^{*}$ & $10^{4}$ & cells $\mathrm{mm}^{-3}$ & 85 & 7 \\
\hline$\delta_{1}$ & $5 \times 10^{-2}$ & day $^{-1}$ & no data found & 7 \\
\hline$\delta_{2}$ & $10^{-3}$ & $\mathrm{~mm}^{3} \mathrm{pg}^{-1}$ day $^{-1}$ & no data found & 7 . \\
\hline$c_{5}$ & 0.42 & day $^{-1}$ & estimated $=c_{1}$ & Appendix $\mathrm{A}$ \\
\hline$k_{3}$ & $1.2 \times 10^{4}$ & $\mathrm{~mm}^{3}$ cells $^{-1}$ & estimated $\approx C^{e q}$ & Appendix $\overline{\mathrm{A}}$ \\
\hline
\end{tabular}

Table 2: A list of parameters appearing in the model equations; those referred to 7 for details are the same as in the ODE model therein presented, while estimation of the newly introduced ones is discussed in Appendix A. Each parameter is supplied with its estimated value, units and source used (when possible) to assess it. References in brackets mean that although the parameter was not directly estimated from a dataset, its calculated value was compared with the biological literature; the caption "no data found" signifies that no suitable data were found to estimate the parameter. Note that $a_{m}$ here corresponds to $a_{M}$ in 7$]$ and $\gamma_{2}$ here to $\gamma$ in 7. $k_{1}^{\text {old }}$ denotes the parameter $k_{1}$ in [7, where it is the macrophage carrying capacity. The parameter $d_{4}$ appears in the boundary conditions for $L$. 
where $\nu \in\{T, M, V, C\}$. For each variable $\nu$ the value of $a_{\nu}$ is chosen to be such that $\nu(0,-\varepsilon)=\nu(0, \ell+\varepsilon)$ is equal to the boundary conditions discussed in the following. Concerning $b$, we will vary its value to see how the "sharpness" of the initial condition will affect lymphangiogenesis. For higher values of $b$, the initial conditions become more step-like and we can interpret this as a deep wound with sharp edges: in this case, there would be (almost) no capillaries in the centre of the wound. On the other hand, assigning smaller values of $b$ would correspond to a shallower initial wound, such that when averaging over the wound depth a certain number of capillaries still remain. As an example, the plot of 20 for $\nu=T$ is shown in Figure 5 for different values of $b$.
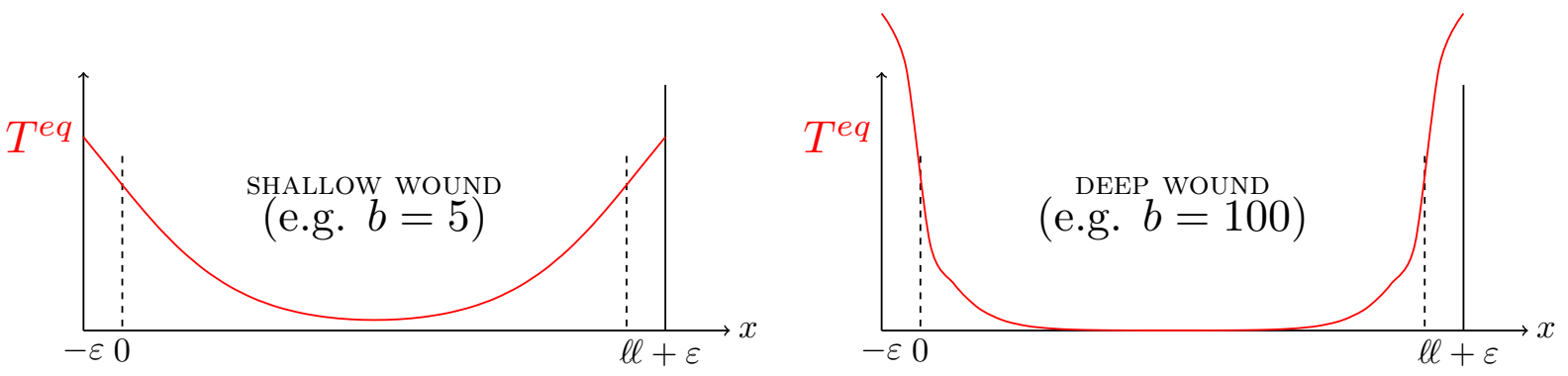

Figure 5: Initial condition $\left.T(0, x)=a_{T} \cdot\{1-[\tanh (b(x-\varepsilon)))+\tanh (b(-x+\ell-\varepsilon))] / 2\right\}$ for different values of b. $T^{e q}$ denotes the $T$-equilibrium level in non-wounded skin.

\section{Boundary Conditions}

First of all, note that boundary conditions are not needed for $C$. We consider Dirichlet boundary conditions for all other variables except $L$, for which we assume Robin boundary conditions. The choice of Dirichlet boundary conditions is dictated by the fact that at the boundary the tissue is in a non-wounded state, and we expect variables to remain close to their normal, equilibrium value there. For $L$, we apply instead the following reasoning.

For LECs, we assume that once they pass the domain edge they move randomly and die at a constant rate $d_{4}$; in fact, it seems unrealistic to assume that they will just vanish once reaching the domain edge. Therefore we will follow common practice for representation of habitat boundaries in ecological modelling [59]: we set a different evolution equation for $L$ inside and outside the domain. In the interior (i.e. for $-\varepsilon<x<\ell+\varepsilon$ ), the dynamics of $L$ will be described by the equation $(13)$; in the exterior (i.e. for $x<-\varepsilon$ and $x>\ell+\varepsilon$ ) instead we assume that LECs move randomly and die (or transdifferentiate) with (high) constant rate $d_{4}$. This gives the equation

$$
\frac{\partial L}{\partial t}=\mu_{L} \frac{\partial^{2} L}{\partial x^{2}}-d_{4} L
$$

outside the wound, whose solution at equilibrium is given by

$$
L_{o}(x)=A_{o} \exp \left(\sqrt{\frac{d_{4}}{\mu_{L}}} x\right)+B_{o} \exp \left(-\sqrt{\frac{d_{4}}{\mu_{L}}} x\right)
$$

where $A_{o}$ and $B_{o}$ are constants. Note that, since we want solutions to be bounded in order to be biologically meaningful, we will take $B_{o}=0$ for $x<-\varepsilon$ and $A_{o}=0$ for $x>\ell+\varepsilon$. Since at the boundaries the outside and the inside solutions should have the same value and the same flux, we have that

$$
\begin{gathered}
\text { at } x=-\varepsilon: \quad L=A_{o} \text { and } \frac{\partial L}{\partial x}=A_{o} \sqrt{\frac{d_{4}}{\mu_{L}}} \Rightarrow \frac{\partial L}{\partial x}(t,-\varepsilon)=\sqrt{\frac{d_{4}}{\mu_{L}}} L(t, 0) \\
\text { at } x=\ell+\varepsilon: \quad L=B_{o} \exp \left(-\sqrt{\frac{d_{4}}{\mu_{L}}} \ell\right) \text { and } \frac{\partial L}{\partial x}=-B_{o} \sqrt{\frac{d_{4}}{\mu_{L}}} \exp \left(-\sqrt{\frac{d_{4}}{\mu_{L}}} \ell\right) \\
\Rightarrow \frac{\partial L}{\partial x}(t, \ell+\varepsilon)=-\sqrt{\frac{d_{4}}{\mu_{L}}} L(t, \ell)
\end{gathered}
$$

which give the boundary conditions for $L$.

Summarising, the boundary conditions are

$$
\begin{aligned}
& \nu(t,-\varepsilon)=\nu(t, \ell+\varepsilon)=\nu^{e q} \quad, \quad E(t,-\varepsilon)=E(t, \ell+\varepsilon)=0, \\
& \frac{\partial L}{\partial x}-\sqrt{\frac{d_{4}}{\mu_{L}}} L=0 \quad \text { at } x=-\varepsilon \quad, \quad \frac{\partial L}{\partial x}+\sqrt{\frac{d_{4}}{\mu_{L}}} L=0 \quad \text { at } x=\ell+\varepsilon
\end{aligned}
$$

with $\nu \in\{T, M, V, C\}$ and where $\nu^{e q}$ denotes the equilibrium value in the unwounded skin for each variable. 


\section{Numerical solutions}

To simulate the two systems (10)-(14) and (15)- 190 , a specific code was written which applies the CrankNicolson method for the diffusion terms and a first-order upwind scheme for the chemotactic terms.

This section is structured as following: first, in 4.1, we present the data sets which will be used as reference points in estimating the "goodness" of the simulations; then, in 4.2 we present a sample simulation of both the whole $\mathrm{O}$ and $\mathrm{S}$ models; in 4.3 we explore how changes in $b$ (initial condition steepness) and $\xi$ (interstitial/lymph flow balance) affect lymphatic regeneration; in 4.4 we address the two extreme cases where there is no advection at all and where the two advection terms sum up (additive advection); finally, in 4.5 we summarise all the observations concerning the different behaviour of $\mathrm{O}$ and $\mathrm{S}$ systems.

\subsection{Data for comparison}

We will compare our model simulations with experimental data reported in Figure 6 These experimental observations show that the overall levels of LECs (both free and in a capillary structure) increase steadily after wounding, and that while at day 10 the vast majority are in the distal half (i.e. upstream the lymph flow) by 60 days they are almost evenly distributed over the two sides.
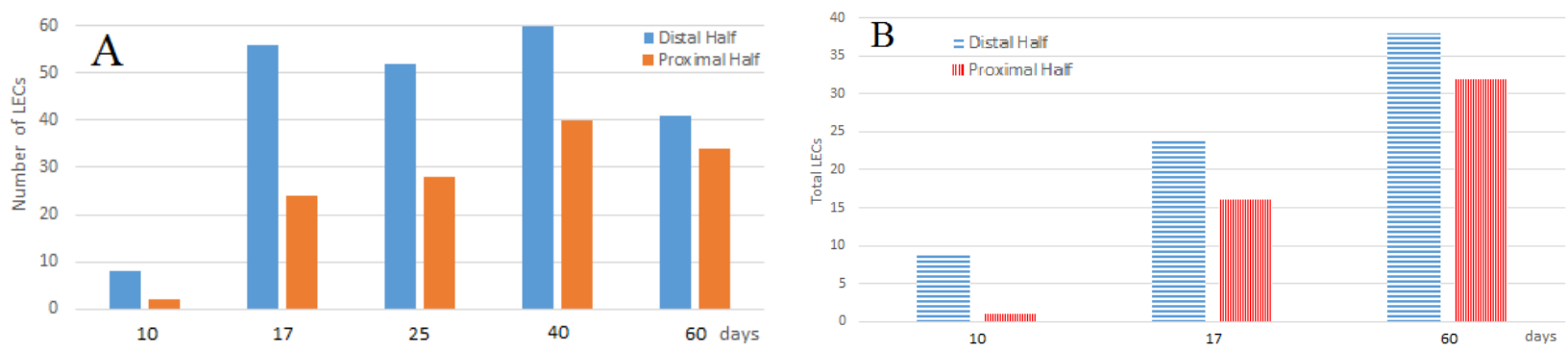

Figure 6: Quantification of LEC presence and distribution in the regenerating region of a mouse tail wound. Here the total numbers of LECs in the distal and proximal halves of the wound at different days post-wounding are reported after data from (A) 85, Figure 2] and (B) 34, Figure 1].

Hence, from experimental data:

- lymphatics should have reached a density close to $C^{e q}$ at day 60 ;

- LEC migration and/or lymphatic capillary formation should happen predominantly in the direction of the lymph/interstitial flow.

\subsection{A first simulation of $\mathrm{O}$ and $\mathrm{S}$}

We start by presenting simulations of the self-organising and sprouting cases (Figures 7 and 8 , respectively) with $\xi=0.5$ (representing that interstitial and lymph flow are equally weighted in the overall advection term) and a very smooth initial condition, with $b=5$ (see (20)).

For these values of $\xi$ and $b$, both systems predict lymphatic regeneration to be almost symmetric and a nearlycomplete network is restored by around day 60 (see Figures 7 and 8 ). Biologically, this represents the situation in which a relatively shallow wound leaves more capillaries in the domain after wounding, so that regeneration occurs mainly from remodelling of the pre-existing network. We note, however, that the distribution of the other variables is highly asymmetric. This will lead to a non-symmetric lymphatic regeneration when parameters are changed so that the chemical concentrations contribute more prominently to the lymphangiogenesis process. One unexpected feature emerging from Figures 7 and 8 is that macrophage, VEGF and LEC levels are higher than equilibrium in the healthy tissue on the right-hand-side of the wound, downstream the lymph flow. While some overspill is likely to be observed, particularly macrophage density appears to be too high to be realistic. In section 4.3 we will present results suggesting that the value $\xi=0.5$ used in Figures 7 and 8 is inappropriately low; the high downstream densities are a consequence of this. However an additional possible explanation might be that more processes are involved in bringing cell and chemical levels back to normal in the healthy skin surrounding a wound; macrophages are likely to be "re-absorbed" in the blood and lymphatic vasculature, where their number is balanced by factors not included in the model. However, the simulations shown in Figures 7 and 8 do predict that eventually all the variables' amounts go back to equilibrium as healing proceeds. 

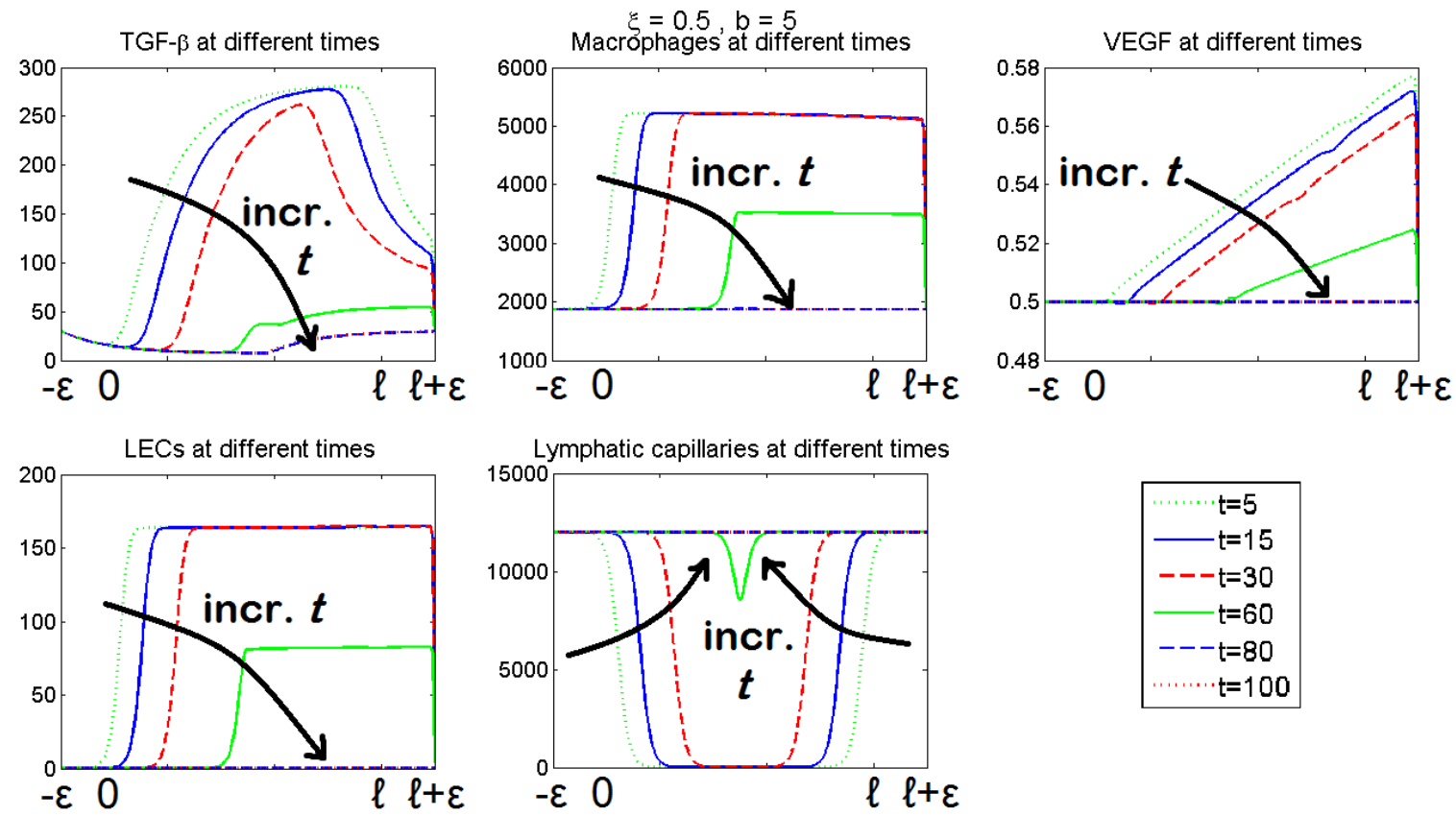

Figure 7: Simulation of equations (10)-(14) (self-organising case) with parameters from Table 2 and initial condition as defined in 3.8 , with $b=5 ; \xi=0.5$. Arrows mark the direction of increasing $t$ in the simulations.
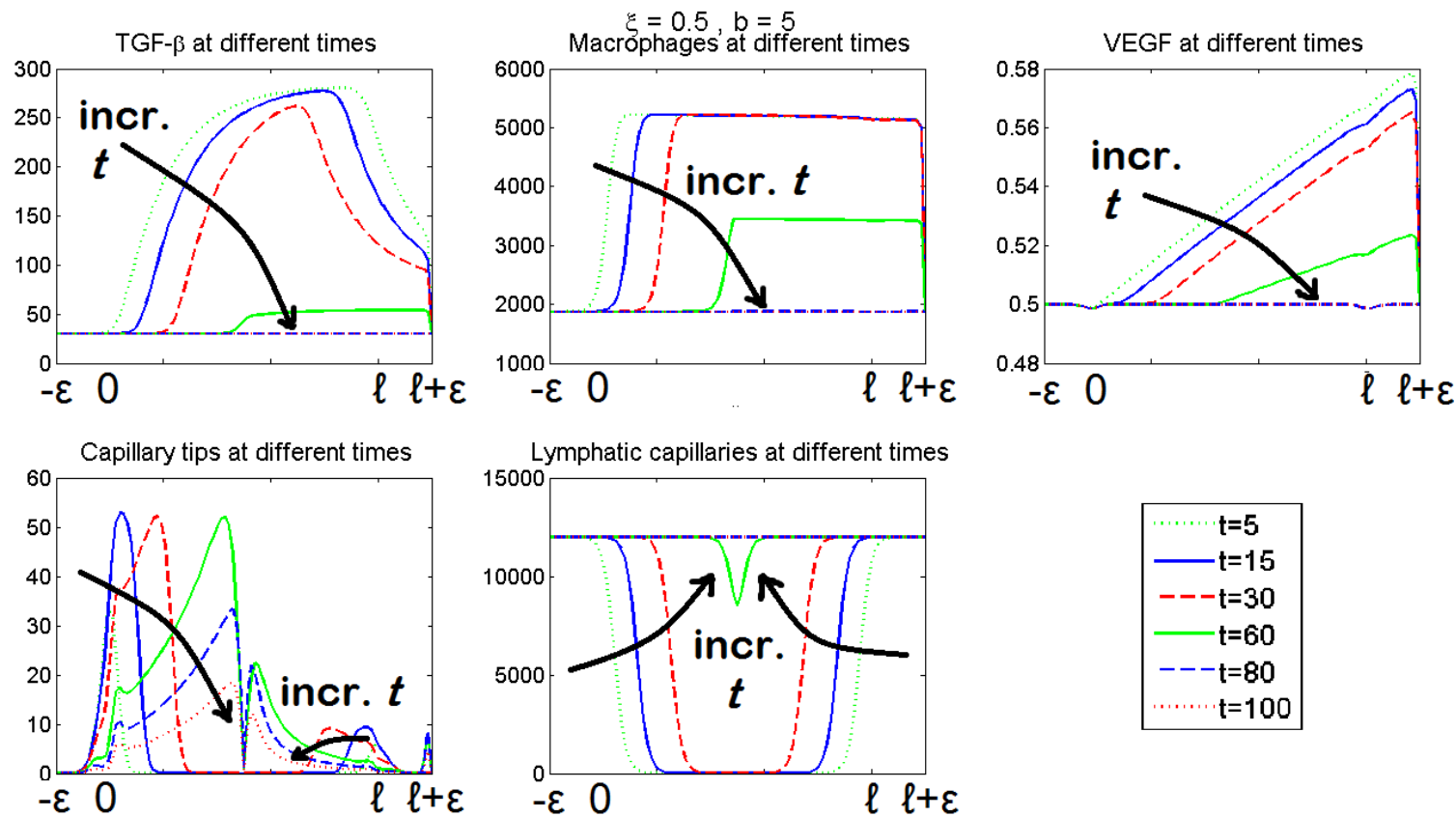

Figure 8: Simulation of equations (15)-(19) (sprouting case) with parameters from Table 2 and initial condition as defined in 3.8 , with $b=5 ; \xi=0.5$. Arrows mark the direction of increasing $t$ in the simulations. 


\subsection{Varying $b$ and $\xi$}

"Visual" observations In order to clearly visualise the changes in dynamics when the parameters $b$ and $\xi$ are varied, we report the approximate solution profiles of the lymphatic capillary density at different times for different combinations of these two parameters; such simulations are reported in Tables 3 and 4 for the self-organising and the sprouting case, respectively.

In the self-organising case, we observe that varying $\xi$ between 0 and 0.75 does not significantly affect the model output for capillary regeneration; on the other hand, the initial conditions play a crucial role, since for a shallow wound $(b=5)$ the lymphatic network is almost completely restored by day 60 , while almost no healing is observed in the deep wound $(b=100)$ scenario. In addition, lymphangiogenesis happens in a fairly symmetric fashion. However, things appear to be quite different for $\xi=1$ : in this case, both shallow and deep wounds exhibit a left-to-right lymphangiogenic process, which is completed by day 60 . Note that while lymphangiogenesis occurs exclusively from left to right in the deep wound scenario, in the shallow wound some lymphatic regeneration is also visible from the right-hand-side of the wound; this confirms our first observation that in a shallow wound logistic remodelling plays a more prominent role than in the deep wound setting. These results suggest that the self-organising hypothesis is supported by the assumption that lymph flow, rather than interstitial flow, is the main contributor to advection in the wound space.

For the sprouting case, things are almost identical to the self-organising case for $0 \leq \xi \leq 0.75$ and $b=5$ (shallow wound scenario). However, varying $\xi$ in this range seems to proportionally increase the left-to-right regeneration speed in the deep wound case $(b=100)$, although it is still unable to account for complete regeneration at day 60 . In addition, for $\xi=1$, while symmetric (although faster) healing is still visible for $b=5$, a capillary front advancing from right to left emerges in the deep wound scenario, though again this is not fast enough to restore the network by day 60. This apparent "switch" of behaviour can be explained as follows. $\xi=1$ corresponds to an advection component due exclusively to lymph flow coming from interrupted capillaries; hence, where $C_{o p}=0$ both cells and chemicals tend to accumulate on one side of the wound. In the self-organising case, however, LECs display random movement and allow the capillary front to move. In the sprouting scenario, on the other hand, capillary tips are not subject to either diffusion or advection; therefore, the front of open capillaries tends to be stuck on the left-hand-side of the wound and chemotaxis tends to happen from right to left. Thus, there is not such an obvious correlation between the value of $\xi$ and the validity of the sprouting hypothesis, in contrast to what we have seen above for the self-organising case. In the sprouting case, a very precise balance of lymph and interstitial flow is required to give a left-to-right lymphangiogenesis which is "fast enough", that is one which completes by day 60 .

To further investigate the "switch" of behaviour (from left-to-right to vice-versa) observed in Table 4 for $b=100$, we run some extra simulations of this case for $0.75<\xi<1$. Results are reported in Figure 9 (note that no significant difference is observed for $0.75<\xi<0.95$, thus we report extra simulations only for values of $\xi$ starting from 0.95). The simulations in Figure 9 suggest that sprouting lymphangiogenesis switches from being left-to-right to being right-to-left as $\xi$ increases from 0 to 1 , passing through symmetrical healing at around $\xi=0.9775$.

Therefore, the most "realistic" value for $\xi$ seems to be $\xi_{O} \approx 1$ for the self-organising case, and $\xi_{S} \approx 0.97$ for the sprouting case. For these values, the self-organising case predicts total healing by day 60 (in accordance with the data discussed in section 4.1, while the sprouting case is a bit delayed in this respect. However, all the variables go back to their equilibrium levels in the latter case, while TGF- $\beta$, macrophages and VEGF stay at a high concentration in the right-hand-side of the wound in the self-organising scenario, which is not what we would expect to happen in reality (simulations not shown).

Quantitative observations In order to make these observations more quantitative and compare them directly with the data sets presented in section 4.1, in each case (i.e. both hypotheses and both combinations of $\xi$ and $b$ values) we calculate a parameter $\pi_{60}$ to quantify the percentage of healing/lymphatic regeneration at day 60. We also count how many LECs are present in the left (distal) and right (proximal) half of the domain at days 10, 17, 25, 40 and 60; in this way, we can directly compare the model output with the empirical data reported in Figure 6 .

To define the quantity $\pi_{60}$, we consider one slice of the wound space, as depicted in Figure 2, we then consider the ratio between the space occupied by the lymphatic capillaries at day 60 and the original wound space. Thus, we consider

$$
\pi_{60}=100 \cdot \frac{S_{C, 60}-S_{I C}}{S_{\text {wound }}}
$$

where $S_{C, 60}$ is calculated as the area under the $C$-curve at $t=60$ (approximated as a polygon using the numerical results shown above) and $S_{\text {wound }}=C^{e q} \cdot(\ell+2 \varepsilon)-S_{I C} ; S_{I C}$ denotes the area subtended by the capillary initial profile curve defined in $[20$, , with $\nu=C$. In this way, we estimate the portion of the real initial wound (i.e. excluding the pre-existing capillary density) occupied by capillaries at day 60 . The values of $\pi_{60}$ for the various cases considered above are reported in Table 5 . 


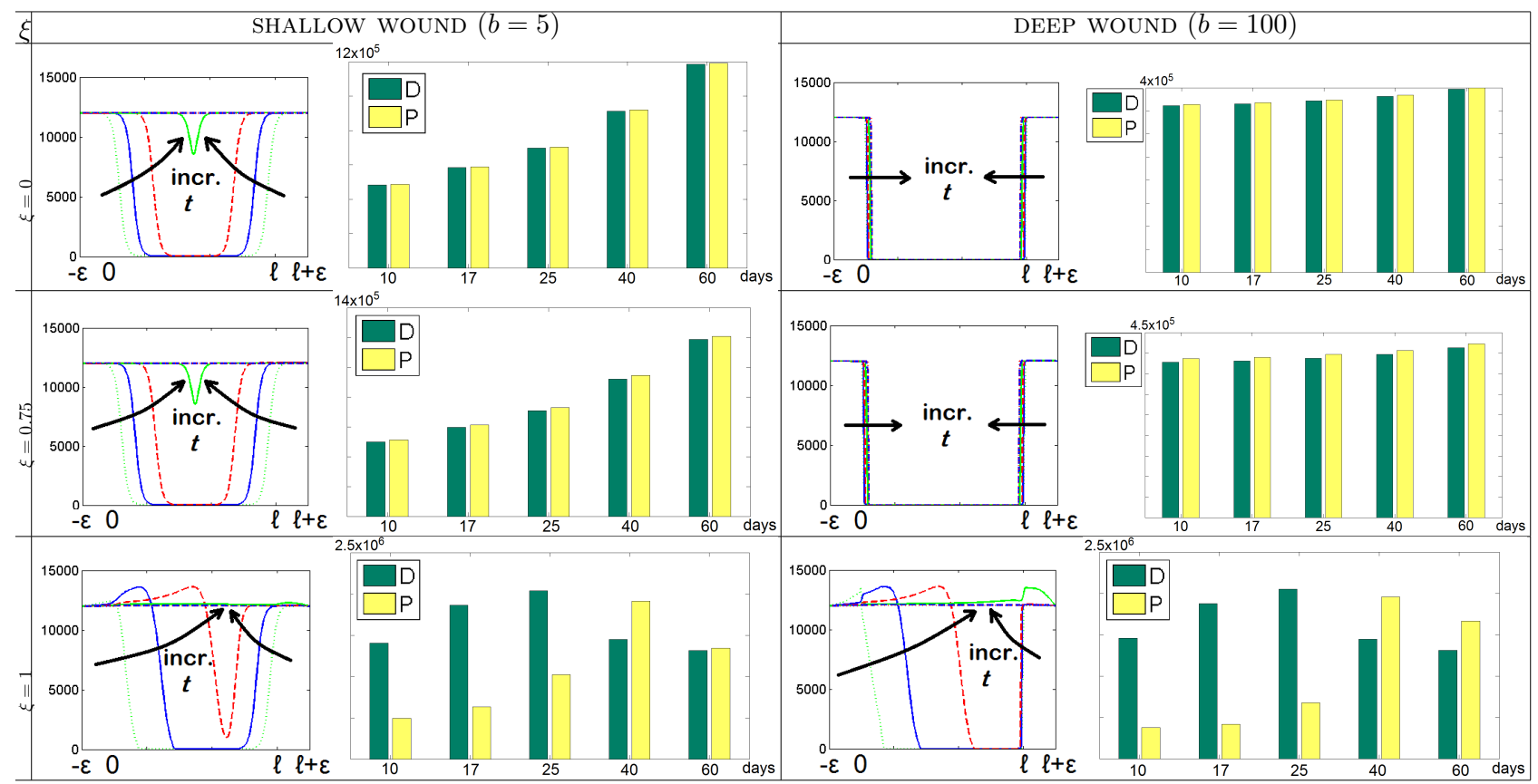

Table 3: Plots of capillary density at different times for different values of $b$ and $\xi$ in the self-organising case (equations (10)-(14)); arrows mark the direction of increasing $t$ in the simulations. On the right-hand-side of each box, we show bar plots of LEC presence (calculated as $L+C$ ) in distal (D) and proximal (P) half of the wound at days 10, 15, 25, 40 and 60 for different values of $b$ and $\xi$.

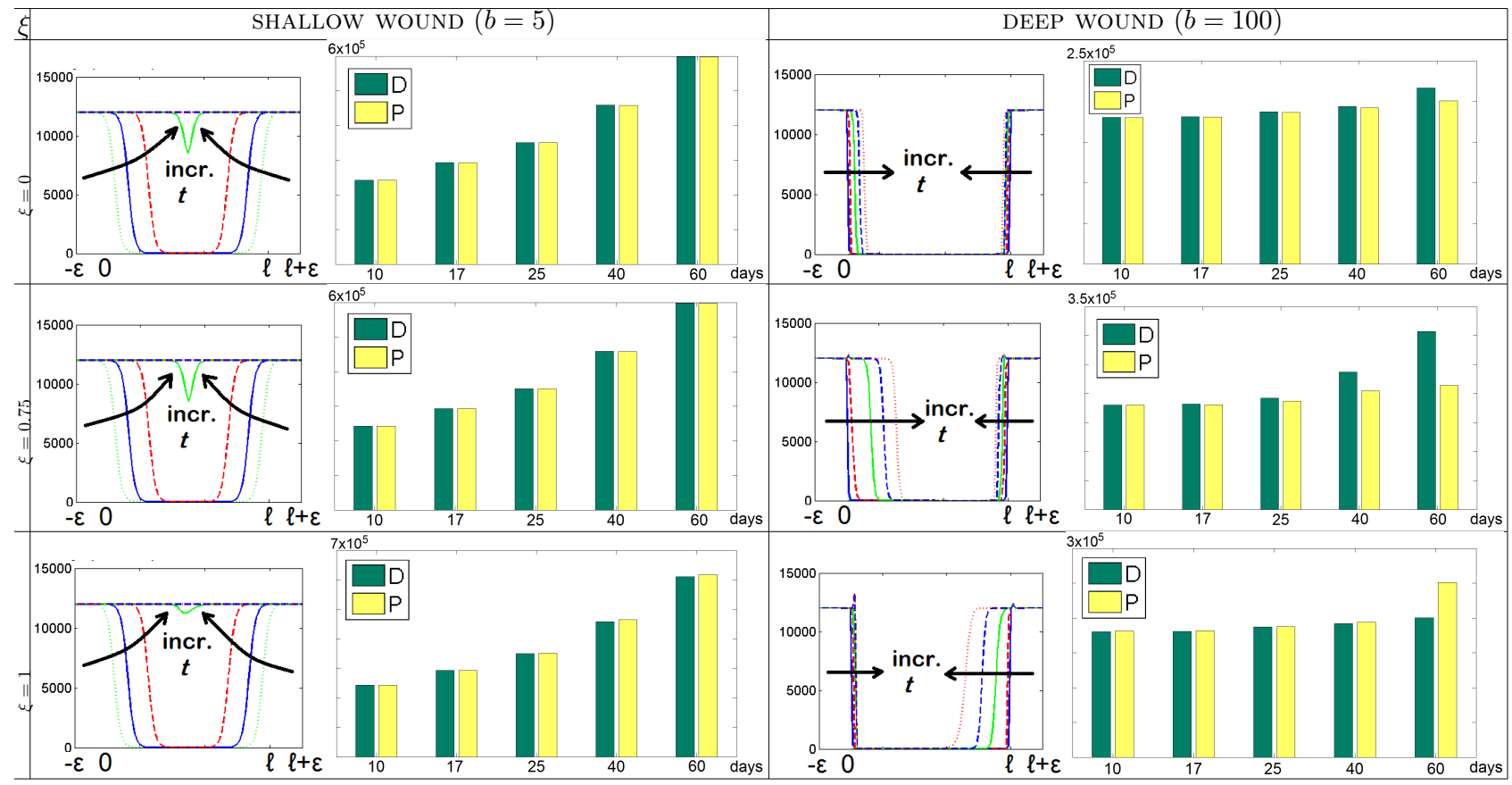

Table 4: Plots of capillary density at different times for different values of $b$ and $\xi$ in the sprouting case (equations (15)-(19)); arrows mark the direction of increasing $t$ in the simulations. On the right-hand-side of each box, we show bar plots of LEC presence (calculated as $E+C$ ) in distal (D) and proximal (P) half of the wound at days 10, 15, 25, 40 and 60 for different values of $b$ and $\xi$. 

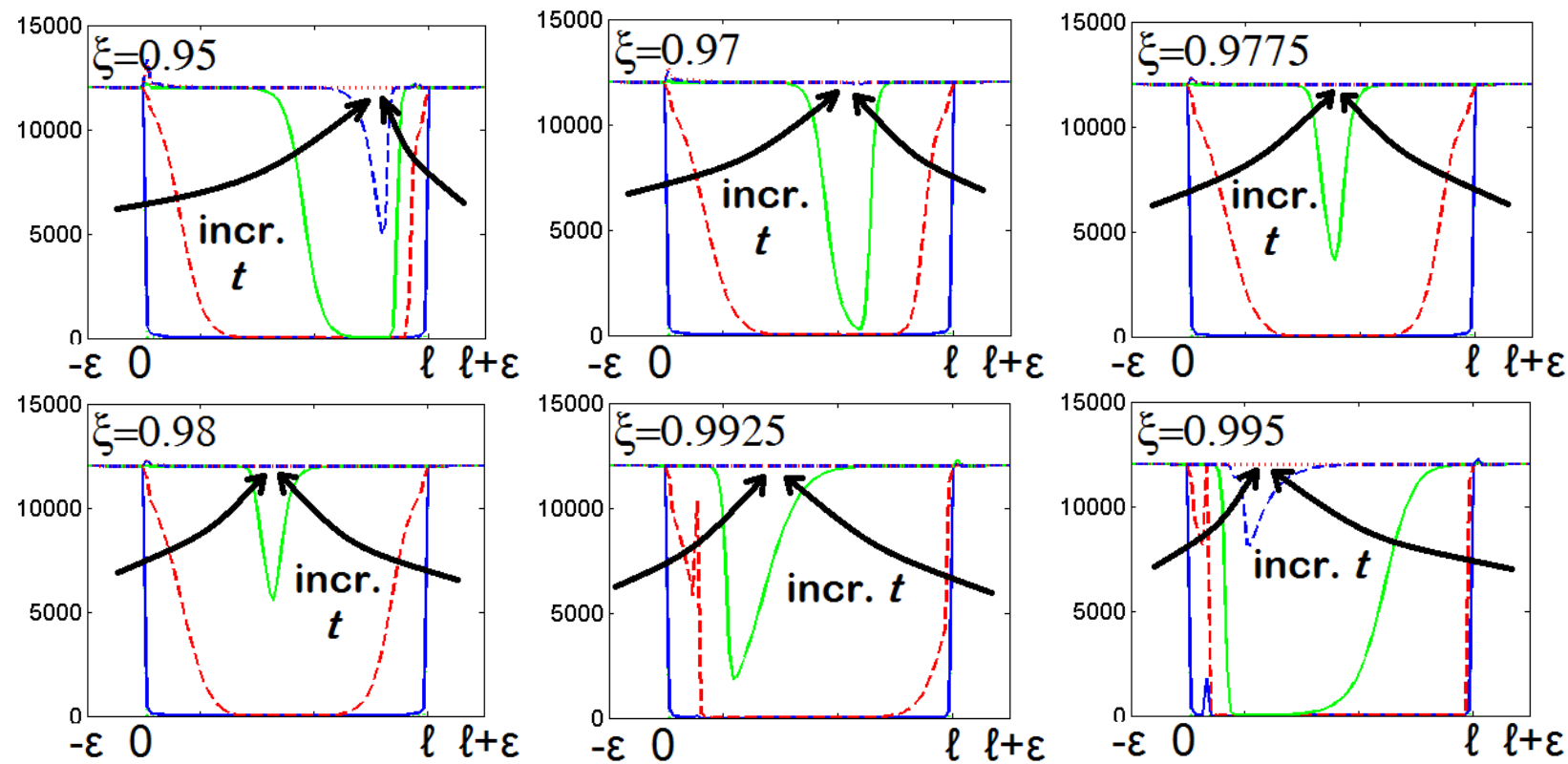

Figure 9: Sprouting case: capillary density dynamics at different times for different values of $\xi$ in the range 0.75 $-1(\xi=0.95,0.97,0.9775,0.98,0.9925,0.995$ - from left to right, top to bottom, respectively), with $b=100$ (deep wound scenario). Arrows mark the direction of increasing $t$ in the simulations.

\begin{tabular}{c|ccccc}
\hline & \multicolumn{5}{|c}{ SHALLOW WOUND $(b=5)$} \\
$\xi$ & 0 & 0.25 & 0.5 & 0.75 & 1 \\
\hline $\mathrm{O}$ & $97.2 \%$ & $97.2 \%$ & $97.2 \%$ & $97.3 \%$ & $101.7 \%$ \\
\hline $\mathrm{S}$ & $97.1 \%$ & $97.1 \%$ & $97.1 \%$ & $97.1 \%$ & $99.2 \%$ \\
\hline \hline & \multicolumn{5}{|c}{ DEEP } \\
\hline & 0 & 0.25 & 0.5 & 0.75 & 1 \\
\hline $\mathrm{O}$ & $5.1 \%$ & $5.1 \%$ & $5.2 \%$ & $5.2 \%$ & $104.1 \%$ \\
$\mathrm{~S}$ & $6.6 \%$ & $8.3 \%$ & $10.5 \%$ & $17.5 \%$ & $10.6 \%$ \\
\hline
\end{tabular}

Table 5: Values of $\pi_{60}$ (defined in (26) ) for different values of $\xi$ and $b$ in the self-organising (O) and sprouting (S) cases. 
From Table 5, we can see clearly how at day 60 the lymphatic vasculature will be restored to a level of $97 \%$ or more for any value of $\xi$ in the shallow wound simulations in both the self-organising and sprouting case. For a deeper wound, however, the lymphatic capillary population is restored only up to about $5 \%$ in the selforganising case and up to about $17 \%$ in the sprouting case for $\xi \leq 0.75$; also, while the parameter $\pi_{60}$ has more or less the same value for all these $\xi$ 's in the self-organising case, we observe an increase in $\pi_{60}$ for increasing $\xi$ in the sprouting scenario (from $6 \%$ to $17 \%$ ). For $\xi=1$, though, the healing predictions are quite different: in the self-organising case, lymphatic capillary density slightly exceeds $100 \%$ healing, while the sprouting case exhibits a capillary regeneration that covers only $10 \%$ of the original wound.

To compare the model predictions with the data reported in Figure 6, we plot the number of LECs (considered as $L+C$ and $E+C$ in the self-organising and sprouting case respectively) in the left (distal) and right (proximal) half of the domain at days 10, 17, 25, 40 and 60. Such numbers are reported as bars in Tables 3 and 4 (righthand-side of each box), which correspond exactly to the cases plotted in Tables 3 and 4 as simulations.

Comparing and contrasting the bar plots reported in Tables 3 and 4 (right-hand-side of each box) with the data sets in Figure 6, we see that the row corresponding to $\xi=1$ is by far the best match for the self-organising case (the other values of $\xi$ giving almost no difference between the distal and proximal LEC density in any day after wounding). For the sprouting case, it is natural to make a different distinction: lymphatic regeneration is always predicted to happen symmetrically in a shallow wound; in a deep wound, a slight distal-biased LEC density is observed appearing at days 40 and 60 for $\xi \leq 0.75$, while for $\xi=1$ the LEC density in the proximal half of the wound overtakes that in the distal half by day 60 .

These observations confirm our first intuition: the self-organising case requires a value of $\xi$ close to one in order to observe a realistically fast left-to-right lymphangiogenesis, while the sprouting hypothesis needs a value of $\xi$ between 0.75 and 1 to produce similarly good results. This difference could be explained by the different mechanisms regulating lymphangiogenesis in each case. In the self-organising hypothesis, capillaries form from LEC self-aggregation and disposition in capillary structures once these are (locally) sufficiently abundant; a constant ever-going interstitial flow slows this down because it prevents local LEC accumulation. In contrast lymph flow occurs only nearby interrupted capillary fronts, which move on as LECs coalesce into vessels. In the sprouting case, by contrast, the total absence of interstitial flow is a problem because neither capillary tips nor well-formed capillaries are subject to either random movement or lymph flow from interrupted capillaries; hence, interstitial flow is the only movement-inducing force, aside from chemical gradients. Moreover, in order to observe a chemical concentration peak on the right-hand-side of the wound (which, by chemotaxis, would induce a left-to-right migration of capillary tips), a good balance is required between an everywhere-present interstitial flow and a locally-active lymph flow.

\subsection{No advection and additive advection cases}

We now consider two final cases: that of no advection at all and that with additive advection (that is, where the advection velocity is as in (1) and (2), but without the coefficients involving the parameter $\xi)$.

Simulations of the no advection case are shown in Table 6 for both the self-organising and sprouting models. Note that, in the self-organising case, dynamics in the absence of advection resemble those reported in Table 3 for $\xi=1$, although here capillary regeneration is a bit slower. Here the driving force behind left-to-right lymphangiogenesis is the influx of macrophages (which produce VEGF) and LECs (which form capillaries) from the left side of the interrupted capillaries. (Recall the influx term from the right edge is zero for macrophages and very small for LECs.)

In the sprouting case, too, capillary density evolution reflects that previously observed for $\xi=1$ (see Table 4 ). However, contrary to the self-organising case, here dynamics are significantly faster in the absence of advection. The reason behind this may lie in macrophages accumulating on the left side on the wound: consequently so does VEGF, which then drives the capillary sprouting from the right towards the peak on the left. Observe that here there are no LECs coming from the open capillaries on the left, so the regeneration is solely directed by gradients (capillary tips move towards increasing gradients of VEGF).

These results suggest two conclusions regarding advection:

1. advection contributes to the speed of the lymphatic regeneration, speeding up the process in the selforganising case and slowing it down under the sprouting hypothesis;

2. advection is of greater importance in the sprouting case, where it actually determines the direction (leftto-right or vice versa) in which healing occurs.

In other words, while the self-organising hypothesis seems to be able to explain left-to-right lymphangiogenesis on its own (thanks to the free LECs influx, primarily from the left side), the sprouting system needs some kind of force pushing VEGF towards the right of the domain so as to form a gradient driving capillary sprouts from the distal to the proximal end of the wound. 


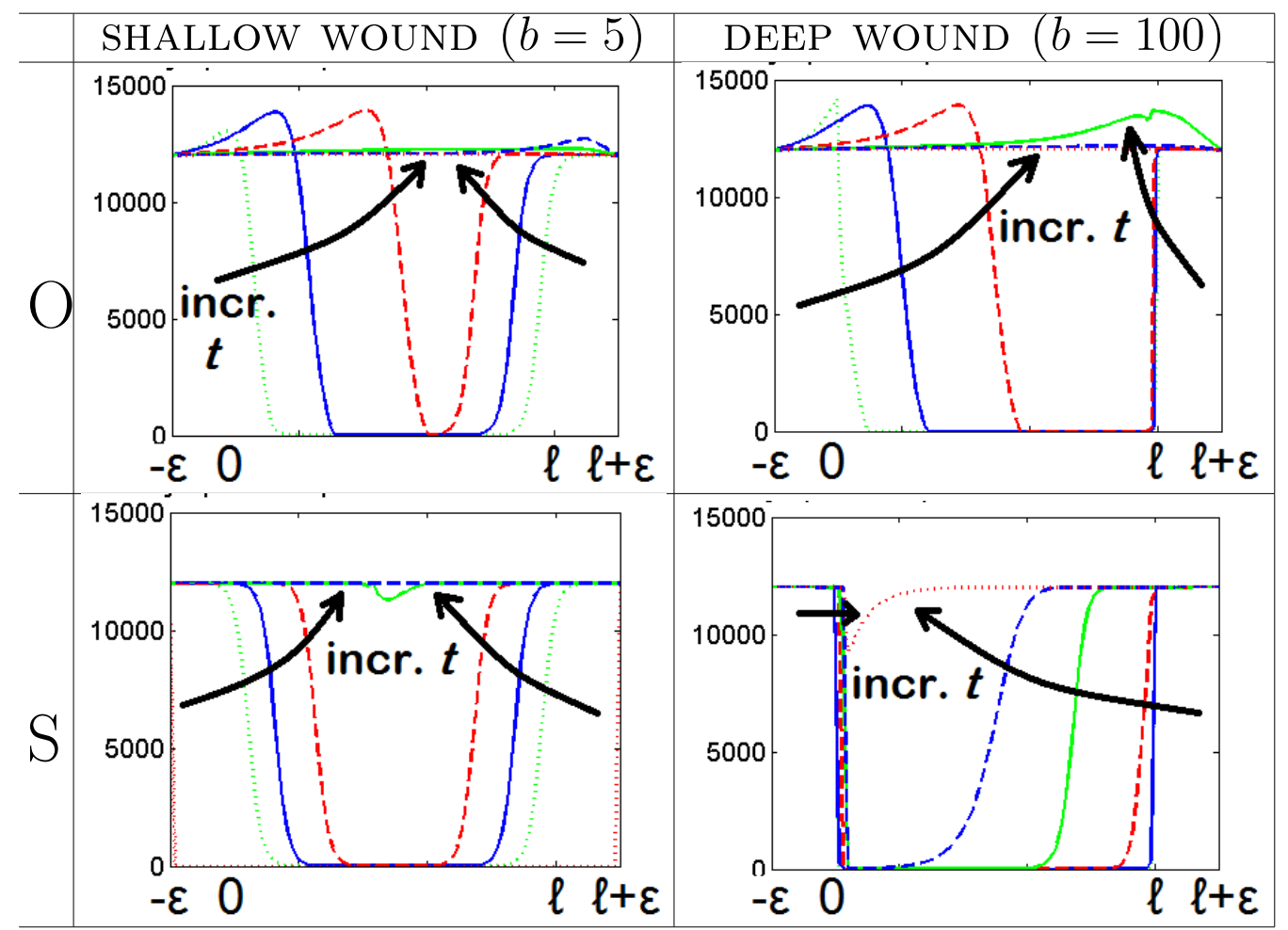

Table 6: Simulation of the self-organising $(\mathrm{O})$ and sprouting $(\mathrm{S})$ systems with parameters from Table 2 and initial condition as defined in 3.8 where the advection terms are switched to zero. Arrows mark the direction of increasing $t$ in the simulations.

Finally, we investigate what happens when the advection velocities for chemicals and cells are replaced, respectively, by

$$
\begin{aligned}
\lambda^{\text {chem }}\left(C_{o p}\right) & =\left(\lambda_{1}^{\text {chem }} \cdot C_{o p}\right)+\lambda_{2}^{\text {chem }} \text { and } \\
\lambda^{\text {cell }}\left(C_{o p}\right) & =\left(\lambda_{1}^{\text {cell }} \cdot C_{o p}\right)+\lambda_{2}^{\text {cell }}
\end{aligned}
$$

we call this the additive advection case. This time lymphangiogenesis, while not appearing overly affected in the shallow wound case, is heavily slowed down in the deep wound scenario (simulation not shown). This reflects the fact that, when lymphatic regeneration is driven mainly by chemical gradients, a sufficiently strong advection force has a negative effect in healing because it does not allow chemicals and cells to accumulate and thereby produce sufficiently steep gradients.

\subsection{Overall comparison of $\mathrm{O}$ and $\mathrm{S}$}

Here the overall similarities and differences between the self-organising and sprouting hypotheses are summarised:

- In shallow wounds lymphangiogenesis appears to be dominated by logistic growth/remodelling and occurs symmetrically from both sides of the wound. In this case, there is little difference between the two hypotheses in terms of the dynamics of wound healing lymphangiogenesis.

- Steeper initial conditions (as in a deep wound) lead to slower capillary regeneration; this is reasonable, since smaller/shallower wounds are expected to heal faster 106, 119 (see also 70 for burn depth). In the deep wound case we also observe a marked difference in behaviour between the two hypotheses: the selforganising case exhibits a very slow progression for values of $\xi$ not close to 1 , with the empirically observed speed occurring for $\xi=1$; by contrast, the sprouting hypothesis predicts lymphangiogenesis to take place from left to right at a speed that increases with $\xi$ up to $\xi \approx 0.9775$, when it becomes symmetric; for larger $\xi$ healing switches to a right-to-left process, at decreasing speed as $\xi$ approaches 1 . This variety of behaviour highlights how important the "balance" between interstitial and lymph flow is in the advection terms (1) and (2). 


\section{Discussion}

The results presented in this paper provide new insights in the understanding of lymphangiogenesis mechanisms. Wound healing lymphangiogenesis is increasingly considered a fundamental aspect of the regeneration process, but there is still no consensus in the scientific community about how this phenomenon takes place. In particular, two main hypotheses have been advanced to describe the lymphangiogenesis process: the selforganising hypothesis [5, 85 and the sprouting hypothesis 74,99 . Here we propose two different PDE systems to describe the two sets of assumptions. The present work shows how the problem of determining the exact lymphatic regeneration mechanism is intertwined with another open question in cellular biology: is interstitial flow a determining factor in cell migration? 86] In this paper we explore the more general case of the effects of advection due to the combination of interstitial flow and lymph flow coming from the interrupted capillaries. In addition, we also consider how different initial conditions, corresponding to shallow and deep wounds, affect the healing process.

The numerical simulations of the two systems we propose as describers for the self-organising and sprouting hypotheses suggest that the observation of left-to-right lymphangiogenesis does not justify per se the selforganising hypothesis: our sprouting-hypothesis system can also reproduce this phenomenon, although for a very precise balance of lymph and interstitial flow. Therefore, a reliable value of $\xi$ is needed in order to choose between the two hypotheses. Other discriminating factors are that:

- capillary density in the sprouting case never significantly exceeds its normal value $C^{e q}$, while overcoming this value is predicted in the self-organising case;

- in the self-organising case there is an excess of TGF- $\beta$, macrophages and VEGF persisting downstream of the lymph flow after capillaries have reached their healthy equilibrium level.

Biologically, it is not clear which is the main contributor to advection between interstitial flow and lymph flow coming from the interrupted capillaries; the models that we have presented suggest the latter is more relevant, and that the value of $\xi$ is above 0.75 in both modelled hypotheses. Moreover, our simulations hint at an inhibiting action of interstitial flow on lymphangiogenesis: strong interstitial flow here seems to significantly slow down capillary regeneration. This may be attributed to the fact that a ubiquitous advection force prevents chemical gradients from forming on the "correct" side of the wound.

Finally, initial conditions (that is, the type of wound, shallow or deep) strongly affect the speed and shape of the regeneration process: deeper wounds require more time to heal, and lymphangiogenesis will occur more markedly in the direction of the lymph flow in this case.

Our results emphasise the importance of advection in tissue regeneration; this concept could be of particular importance in describing the emerging concept of autologous chemotaxis, that is the phenomenon whereby a cell can receive directional cues while at the same time being the source of such cues (see 86, 92]).

Further developments of the model could include the blood vasculature, so to allow a direct comparison between the regenerations of the two vessel structures. The model could also be adapted to investigate differences in lymphatic regeneration in a diabetic scenario, as in 7 . It would also be interesting to investigate the similarities and the differences between wound healing lymphangiogenesis and tumour lymphangiogenesis: tumour cells are known to release lymphangiogenic factors and the tumour mass alters tissue pressure and interstitial flow, which could in turn promote pathological lymphangiogenesis in cancer $15,19,60,83,93$.

A definitive answer to the question of whether the self-organising or sprouting hypothesis better describes lymphangiogenesis will require a more informed evaluation of the relative contribution of interstitial and lymph flow to advection in the wound space, and more detailed spatio-temporal measures of capillary density and chemical concentrations: do we observe a "bump" exceeding normal capillary density along the capillary healing front? Do TGF- $\beta$, macrophages and VEGF persist at a high level downstream of the lymph flow after lymphatic regeneration is complete?

\section{Acknowledgements}

A.B. was funded in part by a Maxwell Institute Scholarship from Heriot-Watt University. K.J.P. acknowledges partial support from BBSRC grant BB/J015940/1. 


\section{A Parameter estimation}

\section{A.1 Sizes, weights, equilibria and velocities}

\section{A.1.1 Domain size}

We consider a full-thickness wound of length $\ell=5 \mathrm{~mm}$, inspired by [117]. For the surrounding skin, we consider a (small) variable width $\varepsilon$. Thus, we have a domain of length $5 \mathrm{~mm}+2 \varepsilon$. In all the simulations reported in the present paper, $\varepsilon=1$; the nature of the observations does not change if a different value of $\varepsilon$ is chosen (simulations not shown).

\section{A.1.2 TGF- $\beta$ molecular weight and equilibrium $T^{e q}$}

We take TGF- $\beta$ molecular weight to be approximately $25 \mathrm{kDa}[9,108$, active/mature isoform]. The equilibrium value of active TGF- $\beta$ is about $30 \mathrm{pg} / \mathrm{mm}^{3}$ [115, Figure 2].

\section{A.1.3 Macrophage volume and equilibrium $M^{e q}$}

A human alveolar macrophage has a volume $V_{M \Phi}$ of approximately $5000 \mu \mathrm{m}^{3}=5 \times 10^{-6} \mathrm{~mm}^{3} 53$. The macrophage steady state can be estimated from [110. Figure 1], which plots typical macrophage density in the skin. This shows that there is an average of about 15 macrophages per $0.1 \mathrm{~mm}^{2}$ field. Assuming a visual depth of $80 \mu \mathrm{m}$, the macrophage density becomes 15 cells $/\left(0.1 \mathrm{~mm}^{2} \times 0.08 \mathrm{~mm}\right)=1875 \mathrm{cells} / \mathrm{mm}^{3}$.

\section{A.1.4 VEGF molecular weight and equilibrium $V^{e q}$}

VEGF molecular weight is taken to be $38 \mathrm{kDa}$ 48, 114, VEGF-165]. The VEGF equilibrium concentration is estimated to be $0.5 \mathrm{pg} / \mathrm{mm}^{3}$ from [42, Figure 1] and [78, Figure 2].

\section{A.1.5 Normal capillary density $C^{e q}$}

In 85] we find that "it was not until day 60, when functional and continuous lymphatic capillaries appeared normal" and "at day 60 the regenerated region had a complete lymphatic vasculature, the morphology of which appeared similar to that of native vessels". Hence, we assume that a capillary network that can be considered "final" appears at day 60, and we take $C^{e q}$ to be the number of LECs present at this time. In [85, Figure 2E] we see that at that time there are about 80 cells. This value corresponds to a $12 \mu \mathrm{m}$ thin section. In addition, from 85 , Figure 2D] we can calculate the observed wound area, which is about $5.6 \times 10^{5} \mu \mathrm{m}^{2}$. In this way we get a volume of $0.0067 \mathrm{~mm}^{3}$ with 80 cells, which corresponds to $C^{e q}=1.2 \times 10^{4}$ cells $/ \mathrm{mm}^{3}$.

\section{A.1.6 Maximum capillary density $C_{\max }$}

First of all, we want to convert 1 capillary section into a cell number. For this purpose, we assume EC crosssectional dimensions to be those reported in [39], namely $10 \mu \mathrm{m} \times 100 \mu \mathrm{m}$. We then assume that LECs lie "longitudinally" along the capillaries, and therefore only the short dimension contributes to cover or "wrap" the circumference of the capillary. Considering a capillary diameter of $55 \mu \mathrm{m}$ as in [28], we have that each lymphatic capillary section is made of approximately 20 LECs (taking into account some overlapping). Then, from 102 we know that EC thickness is approximately $0.5 \mu \mathrm{m}$. Thus a capillary section is a circle of about $55+2 \times 0.5 \mu \mathrm{m}$ diameter, corresponding, as described above, to 20 cells.

If we imagine stacking $1 \mathrm{~mm}^{3}$ with capillaries of this size, we see that we can pile on $1 \mathrm{~mm} / 56 \mu \mathrm{m} \approx 18$ layers of capillaries. Then, considering an EC length of $100 \mu \mathrm{m}$ as in [39], we have that $1 \mathrm{~mm}^{3}$ fits at most a number of capillaries equivalent to the following amount of ECs:

$$
20 \text { cells } \times 18 \times 18 \times \frac{1 \mathrm{~mm}}{100 \mu \mathrm{m}} \approx 6.4 \times 10^{4} \text { cells }=C_{\max } .
$$

\section{A.1.7 Lymph velocity}

28 suggests that the high lymph flow value $(0.51 \mathrm{~mm} / \mathrm{s})$ is due to high pressure following die injection. This suggests that a lower value (9.7 microns/s) might be considered as typical, in agreement with [27]. In both papers the normal lymph velocity seems to be around 10 microns/sec. We thus assume lymph velocity to be $v_{\text {lymph }}=10 \mathrm{micron} / \mathrm{sec}=864 \mathrm{~mm} /$ day $($ from 27,28$)$. 


\section{A.1.8 Interstitial flow velocity}

First of all, we note that in 86 interstitial flow in the skin is calculated to be around 10 microns/sec. (Note that 40 is relevant for this aspect of our modelling, although it is less important for the estimation of parameters; in this reference the synergy between interstitial flow and VEGF gradient is discussed.) Therefore, we will consider the interstitial flow to be also $v_{I F}=10$ microns $/ \mathrm{sec}=864 \mathrm{~mm} /$ day (from [86]).

\section{A.2 Re-calculation of $s_{M}$ and $k_{1}$}

$s_{M}$ here is calculated in the same way as in 7 , but using our amended model equations presented here. For $k_{1}$, we point out that in 77 this parameter was appearing in the logistic part of the $M$-equation: $d M / d t=r_{2} M-r_{2} / k_{1} \cdot M^{2}$. In the PDE systems we do not include such terms because only a minor fraction of macrophages undergo mitosis [38]. However, death due to overcrowding is present in both models; comparing these terms, we see that our "new" $k_{1}$ corresponds to the "old" $k_{1} / r_{2}$.

\section{A.3 Diffusion coefficients}

\section{A.3.1 VEGF diffusion coefficient $D_{V}$}

In 69 the authors observe that "in general, the diffusion coefficient of protein molecules in liquid is of the order of $10^{6} \mu \mathrm{m}^{2} / \mathrm{h}=24 \mathrm{~mm}^{2} /$ day. This intuitively means that a molecule moves $10 \mu \mathrm{m} / \mathrm{sec}$. To generate a gradient over the order of $100 \mu \mathrm{m}$, the timescale of protein decay should be around 10 seconds. In this specific case the protein decay time is about 1-10 hours. Therefore, the observed diffusion coefficient is too large and we need some mechanism to slow down the diffusion" (where "this specific case" means that of VEGF).

In $\left[69\right.$ the VEGF diffusion coefficient is estimated in three different ways: by a theoretical model $\left(0.24 \mathrm{~mm}^{2} /\right.$ day $)$, and by two different empirical techniques $\left(24 \mathrm{~mm}^{2} /\right.$ day $)$. The authors then suggest a diffusion coefficient of the order of $10^{6} \mu \mathrm{m}^{2} / \mathrm{h}=24 \mathrm{~mm}^{2} /$ day. However, they also used the same technique to determine the diffusion coefficient at the cell surface; this time the diffusion coefficient is estimated to be approximately $10^{4} \mu \mathrm{m}^{2} / \mathrm{h}=0.24 \mathrm{~mm}^{2} /$ day. Keeping in mind all these considerations, for the model we take the intermediate value $D_{V}=2.4 \mathrm{~mm}^{2} /$ day.

\section{A.3.2 TGF- $\beta$ diffusion coefficient $D_{T}$}

In 54 the authors estimate a TGF- $\beta$ diffusion coefficient of $0.36 \mathrm{~mm}^{2} / \mathrm{h}=8.64 \mathrm{~mm}^{2} /$ day from 11,35 . In 72 the authors estimate a TGF- $\beta$ diffusion coefficient of $2.54 \mathrm{~mm}^{2} /$ day using the Stokes-Einstein Formula.

We checked their consistency with the estimate for $D_{V}$ above. The Stokes-Einstein equation of these calculated values assumes spherical particles of radius $r$ to have diffusion coefficient $D \sim 1 / r$; since the molecular weight $w$ of a particle is proportional to its volume, we have that $D \sim 1 / \sqrt[3]{w}$ and thus $D_{T} \approx 2.76$.

\section{A.3.3 Macrophage random motility $\mu_{M}$}

In 26 we find "Population random motility was characterized by the random motility coefficient, $\mu$, which was mathematically equivalent to a diffusion coefficient. $\mu$ varied little over a range of C5a [a protein] concentrations with a minimum of $0.86 \times 10^{-8} \mathrm{~cm}^{2} / \mathrm{sec}$ in $1 \times 10^{-7} \mathrm{M} \mathrm{C} 5$ a to a maximum of $1.9 \times 10^{-8} \mathrm{~cm}^{2} / \mathrm{sec}$ in $1 \times 10^{-11} \mathrm{M}$ C5a". We thus take $\mu_{M}$ to be the average of these two values, that is $\mu_{M}=1.38 \times 10^{-8} \mathrm{~cm}^{2} / \mathrm{s} \approx 0.12 \mathrm{~mm}^{2} /$ day.

\section{A.4 Advection parameters $\lambda_{1}$ and $\lambda_{2}$}

We will take $\lambda_{2}^{\text {chem }}$ to be equal to $v_{I F}$ calculated in A.1 thus $\lambda_{2}^{\text {chem }}=864 \mathrm{~mm} /$ day. For $\lambda_{1}^{\text {chem }}$ it is more complicated, but we would say that if $C_{o p}$ reaches the maximum possible value $C_{\max }$ calculated in A.1.6. then $\lambda_{1}^{\text {chem }} \cdot C_{o p}=v_{\text {lymph }}$, which was calculated in A.1. That is, we assume that if the skin is "packed" with open capillaries, then the resulting flow will be the same as the usual lymph flow in the skin lymphatics). Hence $\lambda_{1}^{\text {chem }}=v_{\text {lymph }} / C_{\max }=0.0135 \mathrm{~mm} \mathrm{day}^{-1}$ cell $^{-1}$. For cells we assume smaller values due the higher friction that cells encounter in the tissue. In the absence of relevant empirical data, we take $\lambda_{1}^{\text {cell }}=1 / 10 \cdot \lambda_{1}^{\text {chem }}$ and $\lambda_{2}^{\text {cell }}=1 / 10 \cdot \lambda_{2}^{\text {chem }}$.

\section{A.5 Rate at which TGF- $\beta$ is internalised by macrophages $\gamma_{1}$}

At equilibrium, $C=C^{e q}$ and thus $p(C)=0$. Therefore, the equation for $T$ at equilibrium becomes

$$
a_{M} M^{e q}\left(T_{L}+r_{1} M^{e q}\right)-d_{1} T^{e q}-\gamma_{1} T^{e q} M^{e q}=0,
$$


which leads to

$$
\gamma_{1}=\frac{a_{M} M^{e q}\left(T_{L}+r_{1} M^{e q}\right)-d_{1} T^{e q}}{T^{e q} M^{e q}} \approx 0.0042 \frac{\mathrm{mm}^{3}}{\mathrm{cells} \cdot \text { day }}
$$

\section{A.6 Chemotaxis parameters}

\section{A.6.1 Macrophage chemotactic sensitivity towards TGF- $\beta \chi_{1}$}

In [56, Table 1] the chemotaxis coefficients of neutrophils for different gradients of interleukin- 8 are listed (ranging from $0.6 \times 10^{-7}$ to $\left.12 \times 10^{-7} \mathrm{~mm}^{2} \cdot \mathrm{mL} \cdot \mathrm{ng}^{-1} \cdot \mathrm{s}^{-1}\right)$. We take the intermediate value $\chi_{1}=5 \times 10^{-7} \mathrm{~mm}^{2} \mathrm{~mL} \mathrm{ng}^{-1} \mathrm{~s}^{-1} \approx$ $4 \times 10^{-2} \mathrm{~mm}^{2}\left(\mathrm{pg} / \mathrm{mm}^{3}\right)^{-1}$ day $^{-1}$. To compare this value with one from another source, we consider 101, Figure 8]: although the chemotaxis coefficient is shown to depend on the attractant concentration, an average value is $\chi=150 \mathrm{~cm}^{2} \mathrm{sec}^{-1} \mathrm{M}^{-1} \approx 5.18 \times 10^{-2} \mathrm{~mm}^{2}\left(\mathrm{pg} / \mathrm{mm}^{3}\right)^{-1}$ day $^{-1}$ (using the TGF- $\beta$ molecular weight found in A.1.2. This result is encouraging because it is of the same order of magnitude as the previous estimate.

\section{A.6.2 LEC chemotactic sensitivity towards VEGF $\chi_{2}$}

In [4] a quantification is made of the effects of FGF2 and VEGF165 on HUVEC and HUAEC chemotaxis. In [4, Figure 6A] it is reported that the total distance migrated per HUVEC in response to a $50 \mathrm{ng} / \mathrm{mL}$ gradient of VEGFA165 was about $150 \mu \mathrm{m}$. Considering that the analysed area of the cell migration chamber was $800 \mu \mathrm{m}$ long and that the experiment lasted 200 minutes, we can estimate the endothelial cell velocity to be $150 / 200$ $=0.75 \mu \mathrm{m} / \mathrm{min}=1.08 \mathrm{~mm} /$ day and the VEGF gradient to be $50 \mathrm{ng} / \mathrm{mL} / 800 \mu \mathrm{m}=62.50\left(\mathrm{pg} / \mathrm{mm}^{3}\right) / \mathrm{mm}$. Now, the flux $\mathcal{J}$ in our equation is given by $\mathcal{J}=\chi_{2} L \frac{\partial V}{\partial x}$; however, $\mathcal{J}$ can also be seen as the product of the mass density and the velocity of the flowing mass 23]. Therefore, with $L$ being our mass density, we have

$$
\text { cell velocity }=\chi_{2} \frac{\partial V}{\partial x}
$$

and then we can use the previous calculations to estimate

$$
\chi_{2}=\frac{\text { cell velocity }}{\text { VEGF gradient }}=\frac{1.08 \mathrm{~mm} / \text { day }}{62.50\left(\mathrm{pg} / \mathrm{mm}^{3}\right) / \mathrm{mm}}=0.0173 \frac{\mathrm{mm}^{2}}{\text { day }} \frac{\mathrm{mm}^{3}}{\mathrm{pg}} .
$$

In order to have realistic cell movement dynamics, $\chi_{2}$ is taken to be 10 times bigger. This can be justified by the fact that the aforementioned data refer to HUVECs, and LECs might be faster than these cell types. A more suitable dataset for this parameter would be very useful to better inform this estimate, but we are not aware of such data. Also, chemical gradients created in vitro are usually different between those observed in vivo and they are known to highly affect cell velocity.

\section{A.6.3 Density-dependence of the macrophage chemotactic sensitivity $\omega$}

The cell density-dependence of the macrophage velocity is given by the factor $1 /(1+\omega M)$. This velocity is maximal when $M$ is close to zero and we assume that it is halved when $M$ reaches its carrying capacity $k_{1}^{o l d}$ (that is, the parameter $k_{1}$ in $[7]$ ). We therefore take $\omega$ to be the inverse of the macrophage carrying capacity $k_{1}^{\text {old }}$.

\section{A.7 Macrophage inflow $\phi_{1}$}

We expect $\phi_{1}$ to be proportional to the lymph flow (estimated in A.1 as $v_{\text {lymph }}=864 \mathrm{~mm} \mathrm{day}^{-1}$ ) and macrophage presence in the lymph. In the same source 28 that we used to estimate $v_{l y m p h}$, it is reported that the mean capillary diameter is $55 \mu \mathrm{m}$. Thus about $2.05 \mathrm{~mm}^{3}$ of lymph pass through a capillary bi-dimensional section in 1 day.

In [14] we find that a mouse leukocyte count in the blood is approximately 3 to $8 \times 10^{6}$ cells $/ \mathrm{mL}$, and that of these about $2 \times 10^{6}$ are macrophages coming from the lymph nodes; so we have a macrophage density of $2 \times 10^{3}$ cells $/ \mathrm{mm}^{3}$ in the lymph. Therefore, each day about $2.05 \mathrm{~mm}^{3} \times 2 \times 10^{3}$ cells $/ \mathrm{mm}^{3}=4.11 \times 10^{3}$ macrophages pass in one capillary. Converting capillaries into cell density as was done in A.1.6, we have an influx equal to $\frac{4.11}{20} \times 10^{3}$ day $^{-1}=0.205 \times 10^{3}$ day $^{-1}$. However, the macrophage density reported in 14 refers to blood; we assume that this quantity in lymph (especially during inflammation) will be about 10 times bigger. Therefore, we will take $\phi_{1}=2.05 \times 10^{3}$ day $^{-1}$. 


\section{References}

[1] R. H. Adams and K. Alitalo, Molecular regulation of angiogenesis and lymphangiogenesis, Nat. Rev. Mol. Cell Biol. 8 (2007), no. 6, 464-478.

[2] C. T. Ambrose, Immunology's first priority dispute-an account of the 17th-century Rudbeck-Bartholin feud, Cell. Immunol. 242 (2006), no. 1, 1-8.

[3] J. Asai, H. Takenaka, S. Hirakawa, J. Sakabe, A. Hagura, S. Kishimoto, K. Maruyama, K. Kajiya, S. Kinoshita, Y. Tokura, and N. Katoh, Topical simvastatin accelerates wound healing in diabetes by enhancing angiogenesis and lymphangiogenesis, Am. J. of Pathol. 181 (2012), no. 6, 2217-2224.

[4] I. Barkefors, S. Le Jan, L. Jakobsson, E. Hejll, G. Carlson, H. Johansson, J. Jarvius, J. W. Park, N. Li Jeon, and J. Kreuger, Endothelial cell migration in stable gradients of vascular endothelial growth factor $A$ and fibroblast growth factor 2: effects on chemotaxis and chemokinesis, J. Biol. Chem. 283 (2008), no. 20, 13905-13912.

[5] A. V. Benest, S. J. Harper, S. Y. Herttuala, K. Alitalo, and D. O. Bates, VEGF-C induced angiogenesis preferentially occurs at a distance from lymphangiogenesis, Cardiovasc. Res. 78 (2008), no. 2, 315-323.

[6] P. N. Bernatchez, S. Soker, and M. G. Sirois, Vascular endothelial growth factor effect on endothelial cell proliferation, migration, and platelet-activating factor synthesis is Flk-1-dependent, J. Biol. Chem. 274 (1999), no. 43, 31047-31054.

[7] A. Bianchi, K. J. Painter, and J. A. Sherratt, A mathematical model for lymphangiogenesis in normal and diabetic wounds, Journal of Theoretical Biology 383 (2015), 61-86.

[8] K. C. Boardman and M. A. Swartz, Interstitial flow as a guide for lymphangiogenesis, Circ. Res. 92 (2003), 801-808.

[9] R. Boulton, A. Woodman, D. Calnan, C. Selden, F. Tam, and H. Hodgson, Nonparenchymal cells from regenerating rat liver generate interleukin-1alpha and -1beta: a mechanism of negative regulation of hepatocyte proliferation, Hepatology 26 (1997), no. 1, 49-58.

[10] H. Brem and M. Tomic-Canic, Cellular and molecular basis of wound healing in diabetes, J. Clin. Invest. 117 (2007), no. 5, 1219-1222.

[11] D. R. Brown, Dependence of neurones on astrocytes in a coculture system renders neurones sensitive to transforming growth factor $\beta 1$-induced glutamate toxicity, Journal of Neurochemistry 72 (1999), no. 3, 943-953.

[12] H. M. Byrne and M. A. J. Chaplain, Explicit solutions of a simplified model of capillary sprout growth during tumour angiogenesis, Appl. Math. Lett. 8 (1995), no. 5, 71-76.

[13] H. M. Byrne, M. A. J. Chaplain, D. L. Evans, and I. Hopkinson, Mathematical modelling of angiogenesis in wound healing: Comparison of theory and experiment, J. Theor. Med. 2 (2000), no. 3, 175-197.

[14] C. Cao, D. A. Lawrence, D. K. Strickland, and L. Zhang, A specific role of integrin Mac-1 in accelerated macrophage efflux to the lymphatics, Blood 106 (2005), no. 9, 3234-3241.

[15] Y. Cao, Opinion: emerging mechanisms of tumour lymphangiogenesis and lymphatic metastasis, Nat. Rev. Cancer 5 (2005), no. 9, 735-743.

[16] A. Castiglioni, A history of medicine, Alfred A. Knopf, New York, 1947.

[17] C.-H. Cho, H.-K. Sung, K.-T. Kim, H. G. Cheon, G. T. Oh, H. J. Hong, O.-J. Yoo, and G. Y. Koh, COMP-angiopoietin-1 promotes wound healing through enhanced angiogenesis, lymphangiogenesis, and blood flow in a diabetic mouse model, PNAS 103 (2006), no. 13, 4946-4951.

[18] I. Choi, S. Lee, and Y. K. Hong, The new era of the lymphatic system: no longer secondary to the blood vascular system, Cold Spring Harb. Perspect. Med. 2 (2012), no. 4, a006445.

[19] A. Christiansen and M. Detmar, Lymphangiogenesis and cancer, Genes Cancer 2 (2011), no. 12, 11461158.

[20] C. A. Cobbold and J. A. Sherratt, Mathematical modelling of nitric oxide activity in wound healing can explain keloid and hypertrophic scarring, J. Theor. Biol. 204 (2000), 257-288. 
[21] G. De Crescenzo, S. Grothe, J. Zwaagstra, M. Tsang, and M. D. O'Connor-McCourt, Real-time monitoring of the interactions of transforming growth factor- $\beta$ (TGF- $\beta$ ) isoforms with latency-associated protein and the ectodomains of the TGF- $\beta$ type II and III receptors reveals different kinetic models and stoichiometries of binding, J. Biol. Chem. 276 (2001), no. 32, 29632-29643.

[22] J. F. Dodson, Herophilus of alexandria, Proc. R. Soc. Med. 18 (1924-5), 19-32.

[23] J. F. Douglas, J. M. Gasiorek, J. A. Swaffield, and L. B. Jack, Fluid mechanics, 5th ed., Prentice Hall, 2005.

[24] P. Drew, J. Posnett, and L. Rusling, The cost of wound care for a local population in England, Intl. Wound. J. 4 (2007), no. 2, 149-155.

[25] L. Edelstein, The Propagation of Fungal Colonies: A Model for Tissue Growth, J. Theor. Biol. 98 (1982), 679-701.

[26] B. E. Farrell, R. P. Daniele, and D. A. Lauffenburger, Quantitative relationships between single-cell and cell-population model parameters for chemosensory migration responses of alveolar macrophages to C5a, Cell Motil. Cytoskeleton 16 (1990), no. 4, 279-293.

[27] M. Fischer, U. Costanzo, U. Hoffmann, A. Bollinger, and U. K. Franzeck, Flow velocity of cutaneous lymphatic capillaries in patients with primary lymphedema, Int J Microcirc Clin Exp 17 (1997), no. 3, 143-149.

[28] M. Fischer, U. K. Franzeck, I. Herrig, U. Costanzo, S. Wen, M. Schiesser, U. Hoffmann, and A. Bollinger, Flow velocity of single lymphatic capillaries in human skin, Am. J. Physiol. 270 (1996), no. 1 Pt 2, H358-363.

[29] J. A. Flegg, S. N. Menon, P. K. Maini, and D. L. S. McElwain, On the mathematical modeling of wound healing angiogenesis in skin as a reaction-transport process, Frontiers in Physiology 6 (2015), no. 262.

[30] J. A. Flegg, H. M. Byrne, M. B. Flegg, and D. L. S. McElwain, Wound healing angiogenesis: the clinical implications of a simple mathematical model, J. Theor. Biol. 300 (2012), 309-316.

[31] M. E. Fleury, K. C. Boardman, and M. A. Swartz, Autologous morphogen gradients by subtle interstitial flow and matrix interactions, Biophysical Journal 91 (2006), 113-121.

[32] A. Friedman and G. Lolas, Analysis of a mathematical model of tumor lymphangiogenesis, Math. Models Methods Appl. Sci. 15 (2005), no. 1, 95-107.

[33] P. Galie and R. L. Spilker, A two-dimensional computational model of lymph transport across primary lymphatic valves, J Biomech Eng 131 (2009), no. 11, 111004.

[34] J. Goldman, J. M. Rutkowski, J. D. Shields, M. C. Pasquier, Y. Cui, H. G. Schmokel, S. Willey, D. J. Hicklin, B. Pytowski, and M. A. Swartz, Cooperative and redundant roles of VEGFR-2 and VEGFR-3 signaling in adult lymphangiogenesis, FASEB J. 21 (2007), no. 4, 1003-1012.

[35] G. J. Goodhill, Diffusion in axon guidance, Eur. J. Neurosci. 9 (1997), no. 7, 1414-1421.

[36] A. Gosiewska, C. Yi, O. Blanc-Brude, and J. C. Geesin, Characterization of a macrophage-based system for studying the activation of latent TGF- $\beta$, Meth. Cell Sci. 21 (1999), 47-56.

[37] D. J. Grainger, L. Wakefield, H. W. Bethell, R. W. Farndale, and J. C. Metcalfe, Release and activation of platelet latent TGF-beta in blood clots during dissolution with plasmin, Nat. Med. 1 (1995), no. 9, 932-937.

[38] B. Greenwood, The mitosis of sheep blood monocytes in tissue culture, Quart. J. Exp. Physiol. 58 (1973), $369-377$.

[39] T. L. Haas and B. R. Duling, Morphology favors an endothelial cell pathway for longitudinal conduction within arterioles, Microvasc. Res. 53 (1997), 113-120.

[40] C. L. Helm, M. E. Fleury, A. H. Zisch, F. Boschetti, and M. A. Swartz, Synergy between interstitial flow and VEGF directs capillary morphogenesis in vitro through a gradient amplification mechanism, Proc. Natl. Acad. Sci. U.S.A. 102 (2005), no. 44, 15779-15784.

[41] C. Heppell, T. Roose, and G. Richardson, A model for interstitial drainage through a sliding lymphatic valve, Bull Math Biol 77 (2015), 1101-1131. 
[42] E. Hormbrey, C. Han, A. Roberts, D. A. McGrouther, and A. L. Harris, The relationship of human wound vascular endothelial growth factor (VEGF) after breast cancer surgery to circulating vegf and angiogenesis, Clin. Cancer Res. 9 (2003), 4332-4339.

[43] R. Huggenberger, S. S. Siddiqui, D. Brander, S. Ullmann, K. Zimmermann, M. Antsiferova, S. Werner, K. Alitalo, and M. Detmar, An important role of lymphatic vessel activation in limiting acute inflammation, Blood 117 (2011), no. 17, 4667-4678.

[44] M. Hyytiäinen, C. Penttinen, and J. Keski-Oja, Latent TGF-beta binding proteins: extracellular matrix association and roles in TGF-beta activation, Crit Rev Clin Lab Sci 41 (2004), no. 3, 233-264.

[45] W. J. Jeffcoate and K. G. Harding, Diabetic foot ulcers, Lancet 361 (2003), no. 9368, $1545-1551$.

[46] R. C. Ji, Characteristics of lymphatic endothelial cells in physiological and pathological conditions, Histol. Histopathol. 20 (2005), 155-175.

[47] B. Kaminska, A. Wesolowska, and M. Danilkiewicz, TGF beta signalling and its role in tumor pathogenesis, Acta Biochimica Polonica 52 (2005), no. 2, 329-337.

[48] H. Kaur and L. Y. Yung, Probing high affinity sequences of DNA aptamer against VEGF165, PLoS ONE 7 (2012), no. 2, e31196.

[49] N. Khalil, S. Corne, C. Whitman, and H. Yacyshyn, Plasmin regulates the activation of cell-associated latent TGF-beta 1 secreted by rat alveolar macrophages after in vivo bleomycin injury, Am. J. Respir. Cell Mol. Biol. 15 (1996), no. 2, 252-259.

[50] N. Khalil, C. Whitman, L. Zuo, D. Danielpour, and A. Greenberg, Regulation of alveolar macrophage transforming growth factor- $\beta$ secretion by corticosteroids in bleomycin-induced pulmonary inflammation in the rat, J. Clin. Invest. 92 (1993), 1812-1818.

[51] S. Kiriakidis, E. Andreakos, C. Monaco, B. Foxwell, M. Feldmann, and E. Paleolog, VEGF expression in human macrophages is $N F-\kappa B$-dependent: studies using adenoviruses expressing the endogenous $N F-\kappa B$

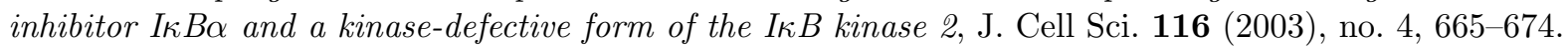

[52] J. Kleinheinz, S. Jung, K. Wermker, C. Fischer, and U. Joos, Release kinetics of VEGF 165 from a collagen matrix and structural matrix changes in a circulation model, Head Face Med. (2010), 6-17.

[53] F. Krombach, S. Munzing, A. M. Allmeling, J. T. Gerlach, J. Behr, and M. Dorger, Cell size of alveolar macrophages: an interspecies comparison, Environ. Health Perspect. 105 Suppl 5 (1997), 1261-1263.

[54] S. Lee, H. J. Hwang, and Y. Kim, Modeling the role of TGF- $\beta$ in regulation of the Th17 phenotype in the LPS-driven immune system, Bull. Math. Biol. 76 (2014), no. 5, 1045-1080.

[55] H. A. Levine, S. Pamuk, B. D. Sleeman, and M. Nilsen-Hamilton, Mathematical modeling of capillary formation and development in tumor angiogenesis: Penetration into the stroma, Bull. Math. Biol. 63 (2001), no. 5, 801-863.

[56] N. Li Jeon, H. Baskaran, S. K. Dertinger, G. M. Whitesides, L. Van de Water, and M. Toner, Neutrophil chemotaxis in linear and complex gradients of interleukin-8 formed in a microfabricated device, Nat. Biotechnol. 20 (2002), no. 8, 826-830.

[57] M. Lohela, M. Bry, T. Tammela, and K. Alitalo, VEGFs and receptors involved in angiogenesis versus lymphangiogenesis, Curr. Opin. Cell Biol. 21 (2009), no. 2, 154-165.

[58] A. Louveau, I. Smirnov, T. J. Keyes, J. D. Eccles, S. J. Rouhani, J. D. Peske, N. C. Derecki, D. Castle, J. W. Mandell, K. S. Lee, T. H. Harris, and J. Kipnis, Structural and functional features of central nervous system lymphatic vessels, Nature 523 (2015), no. 7560, 337-341.

[59] D. Ludwig, D. G. Aronson, and H. F. Weinberger, Spatial Patterning of the Spruce Budworm, J. Math. Biology 8 (1979), 217-258.

[60] S. J. Lunt, T. M. Kalliomaki, A. Brown, V. X. Yang, M. Milosevic, and R. P. Hill, Interstitial fluid pressure, vascularity and metastasis in ectopic, orthotopic and spontaneous tumours, BMC Cancer 8 (2008), 2.

[61] F. Mac Gabhann and A. S. Popel, Model of competitive binding of vascular endothelial growth factor and placental growth factor to VEGF receptors on endothelial cells, Am. J. Physiol. Heart Circ. Physiol. 286 (2004), H153-H164. 
[62] A. J. Macdonald, K. P. Arkill, G. R. Tabor, N. G. McHale, and C. P. Winlove, Modeling flow in collecting lymphatic vessels: one-dimensional flow through a series of contractile elements, Am. J. Physiol. Heart Circ. Physiol. 295 (2008), no. 1, H305-313.

[63] A. Mantovani, A. Sica, S. Sozzani, P. Allavena, A. Vecchi, and M. Locati, The chemokine system in diverse forms of macrophage activation and polarization, Trends Immunol. 25 (2004), no. 12, 677-686.

[64] N. V. Mantzaris, S. Webb, and H. G. Othmer, Mathematical modeling of tumor-induced angiogenesis, J. Math. Biol. 49 (2004), 111-187.

[65] K. N. Margaris and R. A. Black, Modelling the lymphatic system: challenges and opportunities, J. R. Soc. Interf. 9 (2012), no. 69, 601-612.

[66] K. Maruyama, J. Asai, M. Ii, T. Thorne, D. W. Losordo, and P. A. D'Amore, Decreased macrophage number and activation lead to reduced lymphatic vessel formation and contribute to impaired diabetic wound healing, Am. J. Pathol. 70 (2007), 1178-1191.

[67] M. T. G. T. May, Galen on the usefulness of the parts of the body, part ii, Cornell University Press, Ithaca, NY, 1968.

[68] E. Mendoza and G. W. Schmid-Schönbein, A model for mechanics of primary lymphatic valves, J. Biomech. Eng. 125 (2003), 407-414.

[69] T. Miura and R. Tanaka, In vitro vasculogenesis models revisited - measurement of VEGF diffusion in matrigel, Math. Model. Nat. Phenom. 4 (2009), no. 4, 118-130.

[70] S. Monstrey, H. Hoeksema, J. Verbelen, A. Pirayesh, and P. Blondeel, Assessment of burn depth and burn wound healing potential, Burns 34 (2008), 761-769.

[71] G. Müller, J. Behrens, U. Nussbaumer, P. Böhlen, and W. Birchmeier, Inhibitory action of transforming growth factor $\beta$ on endothelial cells, PNAS 84 (1987), 5600-5604.

[72] K. E. Murphy, C. L. Hall, P. K. Maini, S. W. McCue, and D. L. S. McElwain, A fibrocontractive mechanochemical model of dermal wound closure incorporating realistic growth factor kinetics, Bull. Math. Biol. 74 (2012), no. 5, 1143-1170. MR 2909123

[73] V. P. K. H. Nguyen, S. H. Chen, J. Trinh, H. Kim, B. L. Coomber, and D. J. Dumont, Differential response of lymphatic, venous and arterial endothelial cells to angiopoietin-1 and angiopoietin-2, BMC Cell Biol. (2007), 8:10.

[74] C. Norrmen, T. Tammela, T. V. Petrova, and K. Alitalo, Biological basis of therapeutic lymphangiogenesis, Circulation 123 (2011), no. 12, 1335-1351.

[75] I. Nunes, R. L. Shapiro, and D. B. Rifkin, Characterization of latent TGF- $\beta$ activation by murine peritoneal macrophages, J. Immunol. 155 (1995), 1450-1459.

[76] M. Oi, T. Yamamoto, and K. Nishioka, Increased expression of TGF- $\beta 1$ in the sclerotic skin in bleomycin'susceptible' mouse strains, J. Med. Dent. Sci. 51 (2004), 7-17.

[77] G. Oliver and M. Detmar, The rediscovery of the lymphatic system: old and new insights into the development and biological function of the lymphatic vasculature, Genes Dev. 16 (2002), no. 7, 773-783.

[78] A. I. Papaioannou, E. Zakynthinos, K. Kostikas, T. Kiropoulos, A. Koutsokera, A. Ziogas, A. Koutroumpas, L. Sakkas, K. I. Gourgoulianis, and Z. D. Daniil, Serum VEGF levels are related to the presence of pulmonary arterial hypertension in systemic sclerosis, BMC Pulm. Med. (2009), 9:18.

[79] G. F. Pierce, Inflammation in nonhealing diabetic wounds: the space-time continuum does matter, Am. J. Pathol. 159 (2001), no. 2, 399-403.

[80] S. Podgrabinska, P. Braun, P. Velasco, B. Kloos, M. S. Pepper, D. Jackson, and M. Skobe, Molecular characterization of lymphatic endothelial cells, PNAS 99 (2002), no. 25, 16069-16074.

[81] J. Posnett and P. J. Franks, The burden of chronic wounds in the UK, Nurs Times 104 (2008), no. 3, 44-45.

[82] N. P. Reddy and K. Patel, A mathematical model of flow through the terminal lymphatics, Med Eng Phys 17 (1995), no. 2, 134-140. 
[83] E. K. Rofstad, K. Galappathi, and B. S. Mathiesen, Tumor interstitial fluid pressure - A link between tumor hypoxia, microvascular density, and lymph node metastasis, Neoplasia 16 (2014), no. 7, 586-594.

[84] T. Roose and A. C. Fowler, Network development in biological gels: role in lymphatic vessel development, Bull. Math. Biol. 70 (2008), no. 6, 1772-1789.

[85] J. M. Rutkowski, K. C. Boardman, and M. A. Swartz, Characterization of lymphangiogenesis in a model of adult skin regeneration, Am. J. Physiol. Heart. Circ. Physiol. 291 (2006), H1402-H1410.

[86] J. M. Rutkowski and M. A. Swartz, A driving force for change: interstitial flow as a morphoregulator, Trends Cell Biol. 17 (2007), no. 1, 44-50.

[87] A. Saaristo, T. Tammela, A. Farkkila, M. Karkkainen, E. Suominen, S. Yla-Herttuala, and K. Alitalo, Vascular endothelial growth factor-C accelerates diabetic wound healing, Am. J. Pathol. 169 (2006), 10801087.

[88] R. C. Schugart, A. Friedman, R. Zhao, and C. K. Sen, Wound angiogenesis as a function of tissue oxygen tension: a mathematical model, PNAS 105 (2008), no. 7, 2628-2633.

[89] M. Scianna, C. G. Bell, and L. Preziosi, A review of mathematical models for the formation of vascular networks, J. Theor. Biol. 333 (2013), 174-209.

[90] A. Y. Sheikh, J. J. Gibson, M. D. Rollins, H. W. Hopf, Z. Hussain, and T. K. Hunt, Effect of hyperoxia on vascular endothelial growth factor levels in wound model, Arch. Surg. 135 (2000), 1293-1297.

[91] M. Shi, J. Zhu, R. Wang, X. Chen, L. Mi, T. Walz, and T. A. Springer, Latent TGF- $\beta$ structure and activation, Nature 474 (2011), 343-351.

[92] J. D. Shields, M. E. Fleury, C. Yong, A. A. Tomei, G. J. Randolph, and M. A. Swartz, Autologous chemotaxis as a mechanism of tumor cell homing to lymphatics via interstitial flow and autocrine ccr'7 signaling, Cancer Cell 11 (2007), no. 6, 526-538.

[93] T. G. Simonsen, J. V. Gaustad, M. N. Leinaas, and E. K. Rofstad, High interstitial fluid pressure is associated with tumor-line specific vascular abnormalities in human melanoma xenografts, PLoS ONE 7 (2012), no. 6, e40006.

[94] A. J. Singer and R. A. Clark, Cutaneous wound healing, N. Engl. J. Med. 341 (1999), no. 10, 738-746.

[95] W. K. Stadelmann, A. G. Digenis, and G. R. Tobin, Physiology and healing dynamics of chronic cutaneous wounds, Am. J. Surg. 176 (1998), no. 2A Suppl, 26S-38S.

[96] A. B. Sutton, A. E. Canfield, S. L. Schor, M. E. Grant, and A. M. Schor, The response of endothelial cells to TGF $\beta-1$ is dependent upon cell shape, proliferative state and the nature of the substratum, J. Cell Sci. 99 (1991), 777-787.

[97] R. S. Sweat, P. C. Stapor, and W. L. Murfee, Relationships between lymphangiogenesis and angiogenesis during inflammation in rat mesentery microvascular networks, Lymphat. Res. Biol. 10 (2012), no. 4, 198-207.

[98] M. E. Swift, A. L. Burns, K. L. Gray, and L. A. DiPietro, Age-related alterations in the inflammatory response to dermal injury, J. Invest. Dermatol. 117 (2001), no. 5, 1027-1035.

[99] T. Tammela and K. Alitalo, Lymphangiogenesis: Molecular mechanisms and future promise, Cell 140 (2010), 460-476.

[100] A. W. Taylor, Review of the activation of TGF- $\beta$ in immunity, J. Leukocyte Biol. 85 (2009), 29-33.

[101] R. T. Tranquillo, S. H. Zigmond, and D. A. Lauffenburger, Measurement of the chemotaxis coefficient for human neutrophils in the under-agarose migration assay, Cell Motil. Cytoskeleton 11 (1988), no. 1, 1-15.

[102] B. M. van den Berg, H. Vink, and J. A. E. Spaan, The endothelial glycocalyx protects against myocardial edema, Circ. Res. 92 (2003), 592-594.

[103] J. J. L. Velázquez, Point Dynamics in a Singular Limit of the Keller-Segel Model 1: Motion of the Concentration Regions, SIAM J. Appl. Math. 64 (2004), no. 4, 1198-1223.

[104] _ Point Dynamics in a Singular Limit of the Keller-Segel Model 2: Formation of the Concentration Regions, SIAM J. Appl. Math. 64 (2004), no. 4, 1224-1248. 
[105] H. von Staden, Herophilus [and] the art of medicine in early alexandria, Cambridge University Press, Cambridge, UK, 1989.

[106] P. Vowden, Hard-to-heal wounds made easy, Wounds Intern. 2 (2011), no. 4.

[107] S. M. Wahl, D. A. Hunt, L. M. Wakefield, N. McCartney-Francis, L. M. Wahl, A. B. Roberts, and M. B. Sporn, Transforming growth factor type $\beta$ induces monocyte chemotaxis and growth factor production, PNAS 84 (1987), 5788-5792.

[108] L. M. Wakefield, D. M. Smith, K. C. Flanders, and M. B. Sporn, Latent transforming growth factor-beta from human platelets. A high molecular weight complex containing precursor sequences, J. Biol. Chem. 263 (1988), no. 16, 7646-7654.

[109] H. V. Waugh and J. A. Sherratt, Macrophage dynamics in diabetic wound healing, Bull. Math. Biol. 68 (2006), 197-207.

[110] K. Weber-Matthiesen and W. Sterry, Organization of the monocyte/macrophage system of normal human skin, J. Invest. Dermatol. 95 (1990), 83-89.

[111] B. Whitehurst, C. Eversgerd, M. Flister, C. M. Bivens, B. Pickett, D. C. Zawieja, and S. Ran, Molecular profile and proliferative responses of rat lymphatic endothelial cells in culture, Lymph. Res. Biol. 4 (2006), no. 3, 119-142.

[112] E. T. T. Withington, Hippocrates on joints, vol. 3, Harvard University Press, Cambridge, MA, 1984.

[113] M. H. Witte, M. J. Bernas, C. P. Martin, and C. L. Witte, Lymphangiogenesis and lymphangiodysplasia: from molecular to clinical lymphology, Microsc. Res. Tech. 55 (2001), 122-145.

[114] J. P. Yang, H. J. Liu, S. M. Cheng, Z. L. Wang, X. Cheng, H. X. Yu, and X. F. Liu, Direct transport of VEGF from the nasal cavity to brain, Neurosci. Lett. 449 (2009), no. 2, 108-111.

[115] L. Yang, C. X. Qiu, A. Ludlow, M. W. J. Ferguson, and G. Brunner, Active transforming growth factor- $\beta$ in wound repair - determination using a new assay, Am. J. Pathol. 154 (1999), no. 1, 105-111.

[116] I. Zachary and G. Gliki, Signaling transduction mechanisms mediating biological actions of the vascular endothelial growth factor family, Cardiovasc. Res. 49 (2001), 568-581.

[117] Y. Zheng, M. Watanabe, T. Kuraishi, S. Hattori, C. Kai, and M. Shibuya, Chimeric VEGF-ENZ7/PlGF specifically binding to VEGFR-2 accelerates skin wound healing via enhancement of neovascularization, Arterioscler. Thromb. Vasc. Biol. 27 (2007), no. 3, 503-511.

[118] J. C. Zhuang and G. N. Wogan, Growth and viability of macrophages continuously stimulated to produce nitric oxide, PNAS 94 (1997), 11875-11880.

[119] S. Zimny, H. Schatz, and M. Pfohl, Determinants and estimation of healing times in diabetic foot ulcers, J. Diab. Compl. 16 (2002), no. 5, 327-332. 


\section{Contents}

1 Introduction 1

2 Biological background 2

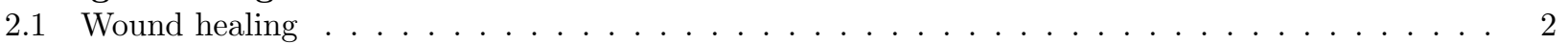

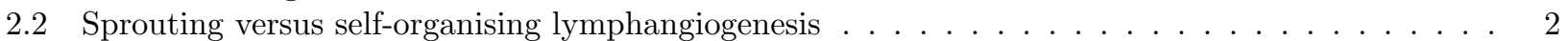

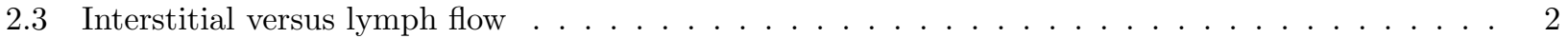

3 Mathematical Modelling 3

3.1 Review of lymphatic-related models $\ldots \ldots \ldots \ldots \ldots \ldots \ldots \ldots \ldots$

3.2 Model targets . . . . . . . . . . . . . . . . . . . . 3

3.3 Model variables and domain $\ldots \ldots \ldots \ldots \ldots \ldots \ldots \ldots \ldots \ldots$



3.5 Self-organising hypothesis $\ldots \ldots \ldots \ldots \ldots \ldots \ldots \ldots \ldots \ldots$

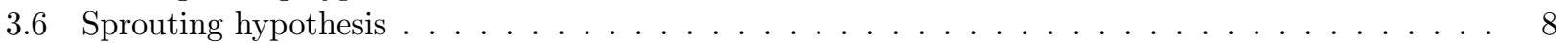

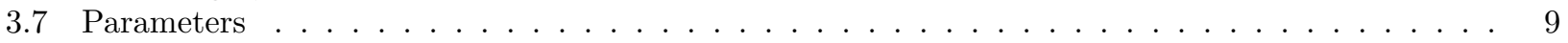

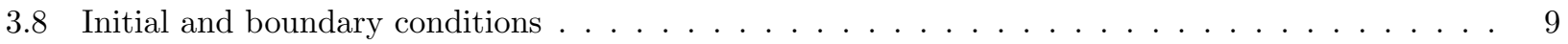

4 Numerical solutions

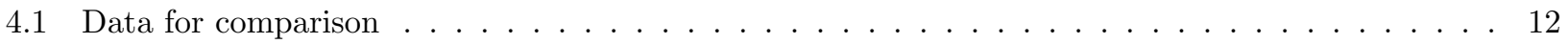

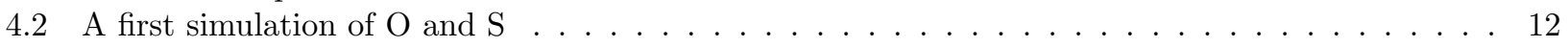

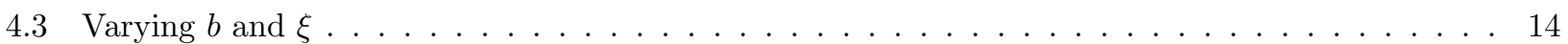

4.4 No advection and additive advection cases . . . . . . . . . . . . . . . . 17

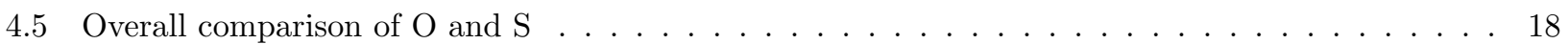

5 Discussion $\quad 19$

A Parameter estimation $\quad \mathbf{2 0}$

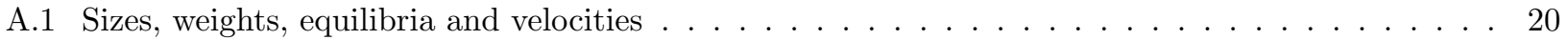

A.1.1 Domain size . . . . . . . . . . . . . . . . . . . . . . . . 20

A.1.2 TGF- $\beta$ molecular weight and equilibrium $T^{e q} \ldots \ldots \ldots \ldots \ldots \ldots \ldots \ldots$

A.1.3 Macrophage volume and equilibrium $M^{e q} \ldots \ldots \ldots \ldots \ldots \ldots \ldots \ldots \ldots$

A.1.4 VEGF molecular weight and equilibrium $V^{e q} \ldots \ldots \ldots \ldots \ldots \ldots \ldots \ldots \ldots$

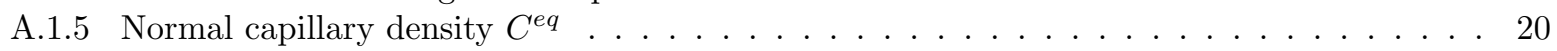

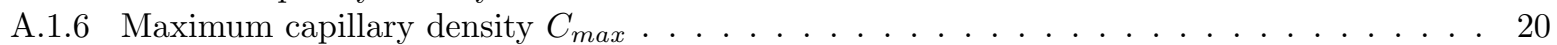

A.1.7 Lymph velocity . . . . . . . . . . . . . . . . . . . . . . . . . 20

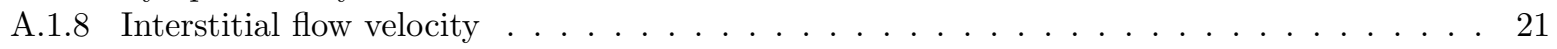

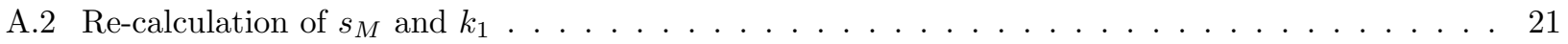

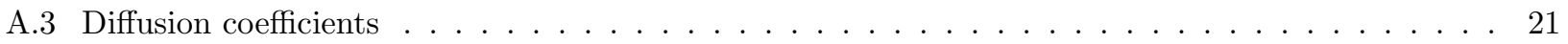

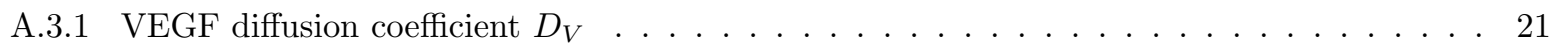

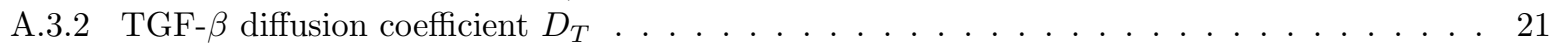

A.3.3 Macrophage random motility $\mu_{M} \ldots \ldots \ldots \ldots \ldots \ldots \ldots \ldots$. . . . . . . . . . . . . .

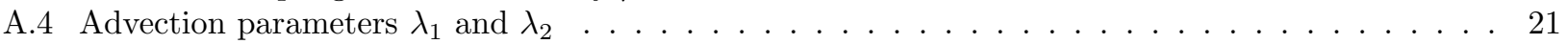

A.5 Rate at which TGF- $\beta$ is internalised by macrophages $\gamma_{1} \ldots \ldots \ldots \ldots \ldots \ldots \ldots$

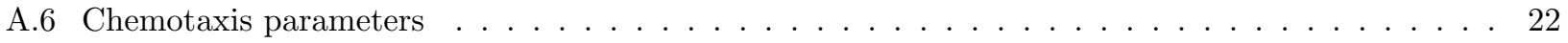

A.6.1 Macrophage chemotactic sensitivity towards TGF- $\beta \chi_{1} \ldots \ldots \ldots \ldots \ldots \ldots$. . . . . .



A.6.3 Density-dependence of the macrophage chemotactic sensitivity $\omega \ldots \ldots \ldots \ldots$. . . . 22

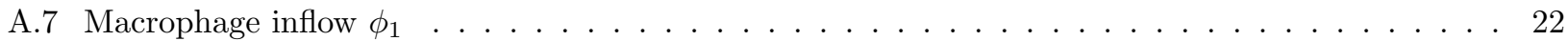

